Egypt. Acad. J. Biolog. Sci., 5(1): 67 - 89 (2013)

Email: egyptianacademic@yahoo.com

Received: 25 / 9 / 2013
C. Physiology \& Molecular Biology

ISSN: 2090-0767

www.eajbs.eg.net

\title{
Effect of lead on some haematological and biochemical characteristics of Clarias gariepinus dietary supplemented with lycopene and vitamin $\mathbf{E}$
}

\author{
Usama M. Mahmoud ${ }^{1}$, Abdel-Basset M.Ebied ${ }^{2}$ and Salwa M. Mohamed ${ }^{2}$ \\ 1- Zoology Department, Faculty of Science, Assiut University \\ 2- Zoology Department, Faculty of Science, South Valley University
}

\begin{abstract}
ASTRACT
The present study aimed to investigate the potential protective effects of lycopene $(9 \mathrm{mg} / \mathrm{kg})$ in comparison with vitamin $\mathrm{E}(50 \mathrm{mg} / \mathrm{kg})$ against the impacts of lead $(\mathrm{Pb})$ toxicity $(7 \mathrm{mg} / \mathrm{l}: 1 / 4$ of $96 \mathrm{~h} \mathrm{LC50})$ on fishes $\mathrm{Pb}$ exposed for 15 and 30 days. $\mathrm{Pb}$ impacts were evaluated in terms of haematological and biochemical characteristics. The activities of aspartate aminotransferase and alanine aminotransferase in serum were significantly increased due to $\mathrm{Pb}$. Treatment with $\mathrm{Pb}$ caused a significant increase in mean corpuscular haemoglobin concentration, serum glucose, total lipid,urea and creatinine and Lipid peroxidation in liver,kidney and gills tissues. On the other hand, $\mathrm{Pb}$ significantly caused decline in serum total protein,albumin, blood haemoglobin, red blood cell count, haematocrit value, mean corpuscular volume and mean corpuscular haemoglobin. It was observed that supplementation of lycopene and vitamin E decreases the toxic effect of lead.
\end{abstract}

Keywords: Fishes, Lead, Lycopene, Vitamin E, Haematological, Biochemical

\section{INTRODUCTION}

Fish are widely used to evaluate the health of aquatic ecosystems because pollutants build up in the food chain and are responsible for adverse effects and death in the aquatic systems (Yousuf and El-Shahawi, 1999; Farkas et al., 2002). The studies carried out on various fishes have shown that heavy metals may alter the physiological activities and biochemical parameters both in tissues and in blood (Tort and Torres, 1988; Canli, 1995; Basa and Usha Rani, 2003). The level of heavy metals in the water and in the sediment of some parts of the river Nile is higher than the tolerance levels or limits set by the Egyptian General Authority for Standards and Quality Control (Anwar, 2003).

Lead $(\mathrm{Pb})$ is one of the most dangerous pollutants in our environment which accumulates in the body due to its low rate of elimination (Harrison and Winchest, 1971). Lead enters aquatic systems from urban, mining and agricultural runoff, atmospheric precipitation, plating process, the use of phosphate fertilizers and gasoline containing lead that leaks from fishery boats and a variety of natural sources, including erosion and volcanic emissions (Denny et al., 1987; Handy, 1994). Several reports have indicated that $\mathrm{Pb}$ can cause neurological, hematological, gastrointestinal, reproductive, circulatory, immunological, histopa-thological and histochemical changes all of them related to the dose and time of exposure to $\mathrm{Pb}$ (Falke and Xwennis, 1990; Royce et al., 1990; Park et al., 2006; Patrick, 2006; Ademuyiwa et al., 2007; Berrahal et al., 2007; Farrag et al., 2007; Abdallah et al., 2010). Also,Lead has many undesired effects, including behavioral (Shafiq-ur-Rehman, 1991), respiratory (Hillam and Ozkan, 1986), visual (Winneke et al., 1988), growth retardation (Shukla et al., 1991), renal (Vyskocil et al., 1989, 1991), hepatic (Honchel et al., 1991; Hao et al., 2002) and reproductive dysfunction (Marchlewicz et al., 1993).and it was 
reported that lead increased the level of lipid peroxidation (Upasani et al., 2001). Lead for example causes renal failure and liver damage (Emmerson, 1973).

Haematological variables are environmentally sensitive and so are used to determine the effects of external stressors and toxic substances (Wendelaar Bonga, 1997). In fish, exposure to chemical pollutants can induce either increases or decreases in haematological levels. Damage to blood and heamapoietic organs in fish may be induced by changes in environmental conditions (Dewilde and Hauston, 1967; Gardner and Yevich, 1969) or water born pollutants (Reichenbach-klink, 1966; Gardner and Yevich, 1969). Blood cell morphology, distribution and indices such as red blood cells (RBCs), hemoglobin $(\mathrm{Hb})$, package cell volume (PCV), mean corpuscular hemoglobin concentration (MCHC), platelets, white blood cells count (WBCs), mean corpuscular volume (MCV), and mean corpuscular hemoglobin $(\mathrm{MCH})$ are good indicators of systemic response to external stress (Srivastava and Choudhay, 2010).

The biochemical parameters in fish are valid for physiopathological evaluation and sensitive for detecting potential adverse effects and relatively early events of pollutant damage (Juneja, and Mahajan, 1983; Ranzani-Paiva et al., 1999; Almeida et al., 2002; Matos et al., 2007; Osman et al., 2010). Many studies have investigated changes in many physiological and biochemical blood indices induced by environmental conditions and the presence of contaminants (Kori-Siakpere, 2006; Maheswaran et al., 2008 and Ololade and Oginni, 2010).

Antioxidants are substances that may protect cells from the damage caused by unstable molecules known as free radicals. Antioxidants interact with and stabilize free radicals and may prevent some of the damage free radicals might otherwise cause. Free radical damage may lead to cancer. Examples of antioxidants include beta-carotene, lycopene, vitamins $\mathrm{C}, \mathrm{E}, \mathrm{A}$ and other substances (Sies, 1997).Vitamin $\mathrm{E}$ is a fat-soluble vitamin that exists in 8 forms. Each form has its own biological activity, which is the measure of potency or functional use in the body (Traber and Packer, 1995). VE has also been shown to play a role in immune function, in DNA repair, and other metabolic process (U.S. Department of Agriculture, 2004). VE functions to protect membrane lipids from damage (Frei, 1991).

Lycopene, a fat soluble carotenoid, is a precursor of b-carotene (Sandmann, 1994) and has at least twice the antioxidant capacity of $\beta$-carotene (Di Mascio et al., 1989). These naturally occurring antioxidants play important roles in animal health by inactivating harmful free radicals produced through normal cellular activity and from various stressors (El-Demerdash et al., 2004).

\section{MATERIALS AND METHODS}

\section{Specimens collection and treatment manipulation}

Eighty healthy fish of The Nile catfish, Clarias gariepinus (200-300 g) in weight, $(33-37 \mathrm{~cm})$ in length were caught from the River Nile at Assiut, Egypt. Fishes immediately were transported to the fish laboratory in the Department of Zoology, Faculty of Science, Assiut University.The experimental fishes were reared in aerated glass tanks $(160 \mathrm{~L}$ capacity) and divided into 8 groups(10 fish /tank) and acclimatized for two weeks before being used in the experimental study. The experimental fish fed pellets at a rate of $3 \%$ of wet weight twice daily. Dead fish were removed and recorded daily .Faeces and residual food were aspirated regularly. The water temperature, $\mathrm{pH}$ and dissolved oxygen (DO) concentration were measured daily $\left(26 \pm .4{ }^{\circ} \mathrm{C}, 7.25 \pm .36 \mathrm{pH}\right.$ and 6.12 $\pm .19 \mathrm{mg} \mathrm{L}-1 \mathrm{DO})$. 
Preparation of tomato paste to adjust the lycopene dose

Tomatoes used for the experiment were obtained from the local market. Fresh peeled, deseeded tomatoes were pulped well to a smooth consistency in a warring blender. The lycopene content in tomato paste was estimated spectrophotometrically according to the methods of Ranganna (1976) and Choudhari and Ananthanarayan (2007). The lycopene concentration in the tomato paste was $30.028 \mathrm{mg} / 100 \mathrm{~g}$. (Okajima et al. 1998). Based on the review of Xianquan et al. (2005), such concentration could not be affected by current conditions of diet preparation and storage of a short time $\left(37^{\circ} \mathrm{C}\right.$ for 4 weeks). In addition to lycopene, tomato paste composition include water, proteins, carbohydrates, fibres, calcium, potassium, zinc, copper, manganese, iron, vitamin $\mathrm{C}$, vitamin $\mathrm{E}$, b-carotenoids and other phytonutrients.

\section{Experimental design}

Fishes were weighed, measured and classified randomly into 8 groups (10 fish/tank) according to dose of lead, tomato paste in terms of lycopene, vitamin $\mathrm{E}$ and their combinations (Table 1). The diets (maize and soy bean, 15 $\mathrm{g} / \mathrm{kg}$ fish) were pelleted after addition of vitamin $\mathrm{E}$ and tomato paste doses for the treated groups and the addition of suitable amounts of molasses and water. The diets were dried at room temperature and stored in small bags for fish feeding.

Table 1: The fish groups exposed to lead $(7 \mathrm{mg} / \mathrm{l})$ and lycopene $(9 \mathrm{mg} / \mathrm{kg}$ body weight) and vitamin $\mathrm{E}$ (50 $\mathrm{mg} / \mathrm{kg}$ body weight) and their combinations.

\begin{tabular}{|l|c|c|c|c|c|c|c|c|}
\hline Treatments & $\mathbf{C}$ & $\mathbf{V E}$ & $\mathbf{L Y C}$ & $\mathbf{L Y C}+\mathbf{V E}$ & $\mathbf{P b}$ & $\mathbf{P b}+\mathbf{V E}$ & $\mathbf{P b}+\mathbf{L Y C}$ & $\mathbf{P b}+\mathbf{L Y C}+\mathbf{V E}$ \\
\hline Lead (mg/L) & 0 & 0 & 0 & 0 & 7 & 7 & 7 & 7 \\
\hline Vitamin E (mg/kg) & 0 & 50 & 0 & 50 & 0 & 50 & 0 & 50 \\
\hline Lycopene (mg/kg) & 0 & 0 & 9 & 9 & 0 & 0 & 9 & 9 \\
\hline
\end{tabular}

$\mathrm{C}=$ control, $\mathrm{VE}=$ vitamin $\mathrm{E}, \mathrm{LYC}=$ lycopene and $\mathrm{Pb}=$ lead dose

Stock solution $(1,000 \mathrm{ppm})$ of lead as lead nitrate $\mathrm{Pb}\left(\mathrm{NO}_{3}\right) 2$ was prepared and stored in clean glass bottles and diluted to concentration of $7 \mathrm{mg} / \mathrm{l}$. Such low sublethal lead concentration (1/4 of $\left.96 \mathrm{~h} \mathrm{LC}_{50}\right)$ was chosen according to levels monitored by Adeyemo et al., (2007). Lead doses were prepared and added constantly to the aquarium for 4 weeks. The test water was replaced daily with the required amount of stock solution to prevent deterioration of water quality and replenish cadmium levels. Tomato paste was added to the diet in concentration of $30 \mathrm{~g} / \mathrm{kg}$ BW $(9 \mathrm{mg}$ lycopene $/ \mathrm{kg}$ BW). Dose response of lycopene was described previously by Rodriguez et al. (2004). Also, vitamin E ( $\alpha$-tocopherol) was supplemented in 50 $\mathrm{mg} / \mathrm{kg}$ BW. Such vitamin E concentration was chosen according to levels monitored by Ortuño et al. (2001). It is worthy to mention that vitamin $\mathrm{E}$ ( $\alpha$ tocopherol) in tomato paste was estimated to be $38.67 \pm 2.29 \mathrm{mg} / 100$ g tomato paste dry weight with no effect by industrial processing (Capanoglu et al., 2008).

\section{Blood analyses}

After 15 and 30 day periods, blood samples of the control and treated fish (4 fish/treatment) were collected from caudal vein of the fish in a small plastic tubes containing heparin solution $(0.2$ $\mathrm{ml} / \mathrm{ml}$ blood) as anticoagulant. These blood samples were used for determining erythrocyte count (Dacie and Lewis 1984) using haemocytometer. Haemoglobin $(\mathrm{Hb})$ was estimated where it was converted into red cyanomethaemoglobin under the influence of potassium ferricyanide and potassium cyanide (Vankampen 1961). Haemoglobin level was determined by using suitable kits (Diamond Diagnostics, Egypt) according to 
Stoskopf (1993). Haematocrit value (Hct) was calculated according to the formulae mentioned by Stoskopf (1993). Mean corpuscular volume (MCV), mean corpuscular haemoglobin (MCH) and mean corpuscular haemoglobin concentration (MCHC) were calculated according to Cloes (1980).

Some other blood samples were collected and left to coagulate for 15-20 min at 4 prior to centrifugation or $20 \mathrm{~min}$ at 3,000 rpm to separate serum. The fresh serum was subjected to biochemical analysis. Serum glucose $(\mathrm{mg} / \mathrm{l})$ was determined, using assay kits supplied by (Spectrum Diagnostics, Egypt). Total protein $(\mathrm{g} / 100 \mathrm{ml})$ content and total lipids (g/l) contents were determined colorimetrically using assay kits supplied by Diamond Diagnostics, Egypt. Activities of aspartate aminotransferase (AST, U/I) and alanine aminotransferase (ALT, U/I) were determined colorimetrically using assay kits (Spectrum Diagnostics, Egypt) according to Reitman and Frankel (1957). The samples were measured by spectrophotometer (Ultroscopec 3100 Pro). Also, serum urea and creatinine were estimated using kits supplied from Biomerieux (France).

\section{Lipid peroxidation measurement}

Lipid peroxidation was indirectly measured according to the method of Ohkawa et al. (1979) (thiobarbituric acid reactive substance, TBARS, test). Ten $(\mathrm{w} / \mathrm{v})$ tissue homogenate from liver,kidney and gills was used (this homogenate contained $1 \% \mathrm{v} / \mathrm{v}$ dimethyl sulfoxide to prevent further oxidation). Tissue homogenate aliquots of $0.2 \mathrm{ml}$ was added to $0.2 \mathrm{ml} 8.1 \% \mathrm{w} / \mathrm{v}$ sodium dodecyl sulphate solution followed by

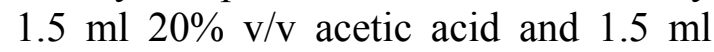
$0.8 \%$ thiobarbituric acid. The mixture was made up to $4.0 \mathrm{ml}$ with distilled water and heated to $95^{\circ} \mathrm{C}$ for $1 \mathrm{~h}$. The samples were cooled, centrifuged at $2,000 \mathrm{rpm}$ for $10 \mathrm{~min}$. and measured at $532 \mathrm{~nm}$ using spectrophotometer
(Ultroscopec 3100 Pro). The results were expressed as nmol malondialdehyde formation per $\mathrm{g}$ tissue.

\section{Statistical analysis}

The basic statistics, means, standard errors and ranges of the measured parameters were estimated. The patterns of variation due to lead, lycopene and vitamin $\mathrm{E}$ doses and their combinations were studied by three- and four-way analysis of variance using the SPSS package (SPSS 1998) at the 0.05 significance level. Levene's test of equality of error variance of the dependent variables was applied, with rejection of the null hypothesis for raw, log-transformed and SQRT-transformed data. So, the homogeneity of variance was assumed for raw data. The pattern of variations was also recorded by one-way analysis of variance, revealing significant difference due to lead, lycopene and vitamin $\mathrm{E}(\mathrm{P} \backslash 0.0001)$; The Tukey-HSD test was considered for multiple comparisons.

\section{RESULTS \\ Haematological parameters}

Haematological parameters the normal values of red blood cell (RBCs) count, haemoglobin content and haematocrit value of C. gariepinus for 15 and 30 days periods are given in Tables (2 \& 3) and Figs. 1-6. The lead and lycopene main effects were highly significant $(\mathrm{P} \backslash 0.0001)$ in both periods for RBCs, $\mathrm{Hb}$ and Hct .No significant main effect of vitamin $E$ was recorded in both periods for $\mathrm{RBCs}, \mathrm{Hb}$ and $\mathrm{Hct}$. $\mathrm{Pb}-\mathrm{VE}$ main effect was highly significant $(\mathrm{P}<0.0001)$ in both periods. Pb-LYC-VE interaction main effect was significant in both exposure periods. The time of exposure main effect was not significant and a positive correlations were recorded between similar RBCs, $\mathrm{Hb}$ and Hct in the two periods ( $\mathrm{r} \mathrm{RBCs}=0.959, \mathrm{r} \mathrm{Hb}=$ 0.961 and $\mathrm{r} \mathrm{Hct}=0.978)$.

The normal value of mean corpuscular value (MCV), mean 
corpuscular haemoglobin $(\mathrm{MCH})$ and mean corpuscular haemoglobin concentration (MCHC) of C. gariepinus in the two periods are given in Tables 2 $\& 3$. The lead main effect was highly significant $(\mathrm{P}<0.0001)$ for $\mathrm{MCV}$ and $\mathrm{MCHC}$ in the two periods. Lycopene main effect was significant only for MCV. No significant main effect of vitamin $\mathrm{E}$ was recorded at both periods for the three previous parameters. $\mathrm{Pb}-\mathrm{VE}$ main effect was significant in both periods for $\mathrm{MCV}$ and for $\mathrm{MCHC}$ at the 15-day period. $\mathrm{Pb}$-LYC main effect was significant in both periods for MCV and for $\mathrm{MCHC}$ at the 30-day period. $\mathrm{Pb}$ LYC-VE interaction showed a significant effect for MCV and MCHC at the 30-day period and for $\mathrm{MCH}$ at the 15-day period. LYC time of exposure effect was not significant in $\mathrm{MCH}$ and $\mathrm{MCHC}$ and significant in MCV.

Table 2: The basic data( $\mathrm{N}=4)$ of blood constituent parameters of $C$. gariepinus exposed to lead $(\mathrm{Pb})$,Vitamin $\mathrm{E}(\mathrm{VE})$, Lycopene (LYC) and their combinations for 15 day.

\begin{tabular}{|c|c|c|c|c|}
\hline Treatments & Control & LYC & $\mathrm{VE}$ & $\mathrm{LYC}+\mathrm{VE}$ \\
\hline Parameters & $\begin{array}{l}\text { Mean } \pm \text { SE } \\
(\text { Min-Max) }\end{array}$ & $\begin{array}{l}\text { Mean } \pm \text { SE } \\
(\text { Min-Max) }\end{array}$ & $\begin{array}{l}\text { Mean } \pm \text { SE } \\
(\text { Min-Max) }\end{array}$ & $\begin{array}{l}\text { Mean } \pm \text { SE } \\
(\text { Min-Max) }\end{array}$ \\
\hline $\mathrm{HB}(\mathrm{g} / 100 \mathrm{ml})$ & $\begin{array}{c}17.08 \pm .34^{\mathrm{C}} \\
(16.46-17.71)\end{array}$ & $\begin{array}{c}18.94 \pm .06^{\mathrm{D}} \\
(18.82-19.08)\end{array}$ & $\begin{array}{c}17.28 \pm .02^{\mathrm{C}} \\
(17.23-17.33)\end{array}$ & $\begin{array}{c}17.3 \pm .19^{\mathrm{C}} \\
(16.94-17.67)\end{array}$ \\
\hline $\begin{array}{c}\mathrm{RBC} \mathrm{x} \\
\left(10^{6} / \mathrm{mm}^{3}\right)\end{array}$ & $\begin{array}{l}2.46 \pm .02^{\mathrm{CD}} \\
(2.41-2.51)\end{array}$ & $\begin{array}{l}2.63 \pm .01^{\mathrm{E}} \\
(2.61-2.66)\end{array}$ & $\begin{array}{l}2.52 \pm .02^{\mathrm{D}} \\
(2.46-2.59)\end{array}$ & $\begin{array}{l}2.47 \pm .02^{\mathrm{D}} \\
(2.42-2.53)\end{array}$ \\
\hline Hct $(\%)$ & $\begin{array}{c}37.31 \pm .15^{\mathrm{C}} \\
(36.09-38.59)\end{array}$ & $\begin{array}{c}42.54 \pm .27^{\mathrm{D}} \\
(42.03-43.05)\end{array}$ & $\begin{array}{l}39.32 \pm .53^{\mathrm{C}} \\
(38.25-40.8)\end{array}$ & $\begin{array}{c}38.36 \pm .53^{\mathrm{C}} \\
(37.24-39.48) \\
\end{array}$ \\
\hline $\operatorname{MCV}\left(\mu \mathrm{m}^{3} /\right.$ cell $)$ & $\begin{array}{c}151.51 \pm .92^{\mathrm{CD}} \\
(149.75-153.74)\end{array}$ & $\begin{array}{c}151.51 \pm 1.19^{\mathrm{E}} \\
(158.27-163.68)\end{array}$ & $\begin{array}{c}155.77 \pm 2.4^{\mathrm{DE}} \\
(150.96-160.62)\end{array}$ & $\begin{array}{l}154.99 \pm 1.79^{\mathrm{DE}} \\
(150.76-159.3)\end{array}$ \\
\hline $\mathrm{MCH}(\mu \mathrm{g} /$ cell $)$ & $\begin{array}{l}69.36 \pm 1.1^{\mathrm{BC}} \\
(66.84-71.78)\end{array}$ & $\begin{array}{c}71.89 \pm .2^{\mathrm{C}} \\
(71.4-72.35)\end{array}$ & $\begin{array}{l}68.45 \pm .72^{\mathrm{AB}} \\
(66.71-70.24)\end{array}$ & $\begin{array}{c}69.92 \pm .8^{\mathrm{BC}} \\
(67.96-71.91) \\
\end{array}$ \\
\hline $\mathrm{MCHC}(\%)$ & $\begin{array}{c}45.77 \pm .54^{\mathrm{AB}} \\
(44.48-47.13)\end{array}$ & $\begin{array}{l}44.53 \pm .32^{\mathrm{AB}} \\
(43.85-45.32)\end{array}$ & $\begin{array}{c}43.95 \pm .53^{\mathrm{A}} \\
(42.47-45.04)\end{array}$ & $\begin{array}{c}45.11 \pm .14^{\mathrm{AB}} \\
(44.75-45.48)\end{array}$ \\
\hline AST & $\begin{array}{c}57.9 \pm 1.27^{\mathrm{A}} \\
(55.34-61.22) \\
\end{array}$ & $\begin{array}{c}87.56 \pm .82^{\mathrm{C}} \\
(85.44-89.16) \\
\end{array}$ & $\begin{array}{c}69.1 \pm 2.59^{\mathrm{AB}} \\
(63.21-74.68) \\
\end{array}$ & $\begin{array}{c}72.27 \pm 1.15^{\mathrm{B}} \\
(69.19-74.14) \\
\end{array}$ \\
\hline ALT & $\begin{array}{l}26.95 \pm .86^{\mathrm{C}} \\
(24.62-28.77)\end{array}$ & $\begin{array}{c}17.91 \pm .25^{\mathrm{A}} \\
(17.22-18.33)\end{array}$ & $\begin{array}{l}24.45 \pm .74^{\mathrm{BC}} \\
(22.75-26.27)\end{array}$ & $\begin{array}{c}21.54 \pm .7^{\mathrm{AB}} \\
(19.63-22.81)\end{array}$ \\
\hline TP & $\begin{array}{l}4.8 \pm .05^{\mathrm{BC}} \\
(4.71-4.95)\end{array}$ & $\begin{array}{l}6.51 \pm .17^{\mathrm{E}} \\
(6.15-6.99)\end{array}$ & $\begin{array}{c}5.8 \pm .1^{\mathrm{DE}} \\
(5.65-6.11)\end{array}$ & $\begin{array}{l}5.93 \pm .03^{\mathrm{DE}} \\
(5.84-5.98)\end{array}$ \\
\hline TL & $\begin{array}{l}9.47 \pm .12^{\mathrm{E}} \\
(9.12-9.66)\end{array}$ & $\begin{array}{c}11.75 \pm .006^{\mathrm{G}} \\
(11.74-11.77)\end{array}$ & $\begin{array}{c}11.42 \pm .1^{\mathrm{FG}} \\
(11.22-11.7)\end{array}$ & $\begin{array}{c}11.12 \pm .04^{\mathrm{F}} \\
(11-11.23)\end{array}$ \\
\hline ALB & $\begin{array}{l}1.7 \pm .06^{\mathrm{CDE}} \\
(1.54-1.86)\end{array}$ & $\begin{array}{l}1.79 \pm .07^{\mathrm{DE}} \\
(1.59-1.93)\end{array}$ & $\begin{array}{l}1.78 \pm .02^{\mathrm{DE}} \\
(1.71-1.85)\end{array}$ & $\begin{array}{c}2.09 \pm .24^{\mathrm{E}} \\
(1.71-2.8)\end{array}$ \\
\hline$\overline{G L U}$ & $\begin{array}{l}85.25 \pm 1.39^{\mathrm{C}} \\
(82.87-88.71)\end{array}$ & $\begin{array}{l}64.62 \pm 1.22{ }^{\mathrm{B}} \\
(61.97-67.19)\end{array}$ & $\begin{array}{c}70.39 \pm 2.23^{\mathrm{B}} \\
(65.14-75.9)\end{array}$ & $\begin{array}{c}52.32 \pm 1.58^{\mathrm{A}} \\
(49.22-55.86)\end{array}$ \\
\hline Urea & $\begin{array}{l}15.42 \pm .08^{\mathrm{AB}} \\
(15.21-15.61)\end{array}$ & $\begin{array}{c}16.33 \pm .08^{\mathrm{B}} \\
(16.11-16.53) \\
\end{array}$ & $\begin{array}{c}18.41 \pm .09^{\mathrm{B}} \\
(18.18-18.65)\end{array}$ & $\begin{array}{l}12.39 \pm .06^{\mathrm{A}} \\
(12.23-12.56 \\
\end{array}$ \\
\hline CREAT & $\begin{array}{l}.44 \pm .01^{\mathrm{B}} \\
(.41-.46)\end{array}$ & $\begin{array}{c}.44 \pm .009^{\mathrm{B}} \\
(.42-.46)\end{array}$ & $\begin{array}{l}.33 \pm .01^{\mathrm{A}} \\
(.32-.36)\end{array}$ & $\begin{array}{l}.41 \pm .01^{\mathrm{B}} \\
(.39-.44)\end{array}$ \\
\hline LPO(K) & $\begin{array}{l}6.75 \pm .01^{\mathrm{D}} \\
(6.71-6.79)\end{array}$ & $\begin{array}{l}3.43 \pm .03^{\mathrm{B}} \\
(3.35-3.51)\end{array}$ & $\begin{array}{l}4.57 \pm .04^{\mathrm{C}} \\
(4.46-4.68)\end{array}$ & $\begin{array}{l}2.74 \pm .07^{\mathrm{A}} \\
(2.55-2.93)\end{array}$ \\
\hline LPO(L) & $\begin{array}{c}5.34 \pm .03^{\mathrm{C}} \\
(5.27-5.41) \\
\end{array}$ & $\begin{array}{c}1.9 \pm .18^{\mathrm{A}} \\
(1.55-2.43)\end{array}$ & $\begin{array}{l}3.18 \pm .01^{\mathrm{B}} \\
(3.15-3.22)\end{array}$ & $\begin{array}{l}1.52 \pm .04^{\mathrm{A}} \\
(1.42-1.64)\end{array}$ \\
\hline$\overline{\mathrm{LPO}(\mathrm{G})}$ & $\begin{array}{c}3.68 \pm .02^{\mathrm{D}} \\
(3.63-3.74)\end{array}$ & $\begin{array}{c}1.57 \pm .02^{\mathrm{B}} \\
(1.52-1.62)\end{array}$ & $\begin{array}{l}2.51 \pm .02^{\mathrm{C}} \\
(2.47-2.56)\end{array}$ & $\begin{array}{c}1.3 \pm .01^{\mathrm{A}} \\
(1.26-1.34)\end{array}$ \\
\hline
\end{tabular}

Different letters indicate significance at $\mathrm{p}<0.05$ Red blood cells (RBCs), Haemoglobin (Hb), Hematocrit (Hct), Mean corpuscular volume (MCV), Mean corpuscular haemoglobin (MCH), Mean corpuscular haemoglobin concentration (MCHC), Aspartate aminotransferase (AST), Alanine aminotransferase (ALT), Total protein (TP), Total lipid (TL), Albumin (Alb), Glucose (GLU), Urea and Creatinine (Creat), Lipid peroxidation in kidney LPO (K), Lipid peroxidation in Liver LPO (L), Lipid peroxidation in Gills LPO (G). 
Table 2: Cont

\begin{tabular}{|c|c|c|c|c|}
\hline Parameters & $\mathrm{Pb}$ & $\mathrm{Pb}+\mathrm{LYC}$ & $\mathrm{Pb}+\mathrm{VE}$ & $\mathrm{Pb}+\mathrm{LYC}+\mathrm{VE}$ \\
\hline & $\begin{array}{l}\text { Mean } \pm \text { SE } \\
\text { (Min-Max) }\end{array}$ & $\begin{array}{l}\text { Mean } \pm \text { SE } \\
\text { (Min-Max) }\end{array}$ & $\begin{array}{l}\text { Mean } \pm \text { SE } \\
\text { (Min-Max) }\end{array}$ & $\begin{array}{l}\text { Mean } \pm \text { SE } \\
\text { (Min-Max) }\end{array}$ \\
\hline $\mathrm{HB}(\mathrm{g} / 100 \mathrm{ml})$ & $\begin{array}{c}14.81 \pm .15^{A} \\
(14.51-15.11)\end{array}$ & $\begin{array}{c}16.28 \pm . .18^{\mathrm{B}} \\
(15.94-16.63)\end{array}$ & $\begin{array}{c}15.37 \pm .06^{A} \\
(15.23-15.51)\end{array}$ & $\begin{array}{l}15.31 \pm .05^{A} \\
(15.2-15.42)\end{array}$ \\
\hline $\begin{array}{c}\mathrm{RBC} \mathrm{x} \\
\left(10^{6} / \mathrm{mm}^{3}\right)\end{array}$ & $\begin{array}{l}2.15 \pm .02^{\mathrm{A}} \\
(2.1-2.22)\end{array}$ & $\begin{array}{l}2.37 \pm .02^{\mathrm{BC}} \\
(2.31-2.43)\end{array}$ & $\begin{array}{l}2.19 \pm .01^{\mathrm{A}} \\
(2.16-2.23)\end{array}$ & $\begin{array}{l}2.31 \pm .006^{\mathrm{B}} \\
(2.3-2.33)\end{array}$ \\
\hline Hct $(\%)$ & $\begin{array}{l}28.83 \pm .68^{\mathrm{A}} \\
(27.46-30.2)\end{array}$ & $\begin{array}{c}34.63 \pm .63^{\mathrm{B}} \\
(33.21-36.07)\end{array}$ & $\begin{array}{c}30.7 \pm .51^{\mathrm{A}} \\
(29.7-32.02)\end{array}$ & $\begin{array}{l}33.6 \pm .05^{\mathrm{B}} \\
(33.5-33.7)\end{array}$ \\
\hline $\operatorname{MCV}\left(\mu \mathrm{m}^{3} /\right.$ cell $)$ & $\begin{array}{c}133.64 \pm 2.93^{\mathrm{A}} \\
(127.21-140.56)\end{array}$ & $\begin{array}{l}146.12 \pm 2.03^{\mathrm{BC}} \\
(141.75-150.55)\end{array}$ & $\begin{array}{l}139.87 \pm 1.62^{\mathrm{AB}} \\
(136.81-143.58)\end{array}$ & $\begin{array}{c}145.16 \pm .48^{\mathrm{BC}} \\
(143.77-145.88)\end{array}$ \\
\hline $\mathrm{MCH}(\mu \mathrm{g} /$ cell $)$ & $\begin{array}{l}68.66 \pm .95^{\mathrm{AB}} \\
(66.48-71.03)\end{array}$ & $\begin{array}{l}68.71 \pm .19^{\mathrm{AB}} \\
(68.33-69.08)\end{array}$ & $\begin{array}{c}70.02 \pm .21^{\mathrm{BC}} \\
(69.55-70.5)\end{array}$ & $\begin{array}{c}66.13 \pm .21^{\mathrm{A}} \\
(65.73-66.75)\end{array}$ \\
\hline $\mathrm{MCHC}(\%)$ & $\begin{array}{c}51.41 \pm .67^{\mathrm{C}} \\
(50.03-52.84)\end{array}$ & $\begin{array}{c}47.05 \pm .78^{\mathrm{B}} \\
(45.38-48.73)\end{array}$ & $\begin{array}{c}50.08 \pm .73^{\mathrm{C}} \\
(48.43-51.36)\end{array}$ & $\begin{array}{c}45.55 \pm .15^{\mathrm{AB}} \\
(45.26-45.88)\end{array}$ \\
\hline AST & $\begin{array}{c}191.05 \pm .91^{\mathrm{F}} \\
(188.4-192.33)\end{array}$ & $\begin{array}{c}149.14 \pm 2.39^{\mathrm{E}} \\
(142.51-153.65)\end{array}$ & $\begin{array}{c}132.14 \pm 5.08^{\mathrm{D}} \\
(121.38-142.66)\end{array}$ & $\begin{array}{c}127.22 \pm 4.54^{\mathrm{D}} \\
(113.92-134.18)\end{array}$ \\
\hline ALT & $\begin{array}{c}49.9 \pm 1.62^{\mathrm{E}} \\
(45.38-53.01)\end{array}$ & $\begin{array}{c}34.98 \pm .96^{\mathrm{D}} \\
(33.45-37.87)\end{array}$ & $\begin{array}{l}38.34 \pm 1.22^{\mathrm{D}} \\
(35.08-40.49)\end{array}$ & $\begin{array}{c}34.07 \pm .68^{\mathrm{D}} \\
(32.44-35.22)\end{array}$ \\
\hline TP & $\begin{array}{l}3.27 \pm .13^{\mathrm{A}} \\
(3.09-3.67)\end{array}$ & $\begin{array}{c}5.05 \pm .09^{\mathrm{CD}} \\
(4.88-5.3)\end{array}$ & $\begin{array}{l}4.5 \pm .31^{\mathrm{BC}} \\
(3.87-5.31)\end{array}$ & $\begin{array}{l}4.23 \pm 0.1^{\mathrm{B}} \\
(4.03-4.49)\end{array}$ \\
\hline TL & $\begin{array}{l}5.54 \pm .16^{\mathrm{A}} \\
(5.11-5.91)\end{array}$ & $\begin{array}{l}8.36 \pm .09^{\mathrm{D}} \\
(8.21-8.57)\end{array}$ & $\begin{array}{l}6.92 \pm .1^{\mathrm{B}} \\
(6.75-7.18)\end{array}$ & $\begin{array}{l}7.55 \pm .01^{\mathrm{C}} \\
(7.51-7.59)\end{array}$ \\
\hline ALB & $\begin{array}{c}.98 \pm .009^{\mathrm{A}} \\
(.96-1)\end{array}$ & $\begin{array}{l}1.24 \pm .02^{\mathrm{AB}} \\
(1.17-1.28)\end{array}$ & $\begin{array}{c}1.29 \pm .02^{\mathrm{ABC}} \\
(1.23-1.35)\end{array}$ & $\begin{array}{c}1.59 \pm .01^{\mathrm{BCD}} \\
(1.55-1.63)\end{array}$ \\
\hline GLU & $\begin{array}{c}117.62 \pm 1.98^{\mathrm{E}} \\
(112.03-121.24)\end{array}$ & $\begin{array}{l}102.05 \pm 1.46^{\mathrm{D}} \\
(99.4-105.44)\end{array}$ & $\begin{array}{c}107.71 \pm 1.73^{\mathrm{D}} \\
(103.5-112.01)\end{array}$ & $\begin{array}{c}99.78 \pm 2.48^{\mathrm{D}} \\
(93.66-105.22)\end{array}$ \\
\hline Urea & $\begin{array}{l}29.79 \pm 1.13^{\mathrm{D}} \\
(27.15-32.61)\end{array}$ & $\begin{array}{l}32.05 \pm 1.21^{\mathrm{D}} \\
(29.13-34.97)\end{array}$ & $\begin{array}{c}29.1 \pm 1.1^{\mathrm{D}} \\
(26.17-31.43)\end{array}$ & $\begin{array}{c}24.42 \pm 1.007^{\mathrm{C}} \\
(22.23-26.83)\end{array}$ \\
\hline CREAT & $\begin{array}{c}.87 \pm .009^{\mathrm{D}} \\
(.85-.89)\end{array}$ & $\begin{array}{r}.83 \pm .009^{\mathrm{D}} \\
(.81-.85)\end{array}$ & $\begin{array}{l}.65 \pm .01^{\mathrm{C}} \\
(.63-.68)\end{array}$ & $\begin{array}{r}.62 \pm .01^{\mathrm{C}} \\
(.6-.64)\end{array}$ \\
\hline $\mathrm{LPO}(\mathrm{K})$ & $\begin{array}{c}49.25 \pm 1.85^{\mathrm{F}} \\
(46-54.5)\end{array}$ & $\begin{array}{c}12.05 \pm .5^{\mathrm{D}} \\
(10.85-13.3)\end{array}$ & $\begin{array}{l}16.83 \pm .47^{\mathrm{E}} \\
(16.15-18.2)\end{array}$ & $\begin{array}{l}6.87 \pm .3^{\mathrm{C}} \\
(6.4-7.75)\end{array}$ \\
\hline LPO(L) & $\begin{array}{l}26.52 \pm .82^{\mathrm{E}} \\
(25.05-28.5)\end{array}$ & $\begin{array}{l}7.86 \pm .62^{\mathrm{C}} \\
(6.6-9.45)\end{array}$ & $\begin{array}{l}13.12 \pm .34^{\mathrm{D}} \\
(12.15-13.6)\end{array}$ & $\begin{array}{l}5.86 \pm .29^{\mathrm{C}} \\
(5.2-6.55)\end{array}$ \\
\hline LPO(G) & $\begin{array}{l}24.37 \pm .9^{\mathrm{D}} \\
(23.05-27)\end{array}$ & $\begin{array}{l}6.57 \pm .61^{\mathrm{C}} \\
(5.1-7.9)\end{array}$ & $\begin{array}{r}8.83 \pm .82^{\mathrm{C}} \\
(7.3-11)\end{array}$ & $\begin{array}{l}2.8 \pm .05^{\mathrm{B}} \\
(2.65-2.9)\end{array}$ \\
\hline
\end{tabular}

Different letters indicate significance at $\mathrm{p}<0.05$ Red blood cells (RBCs), Haemoglobin ( $\mathrm{Hb}$ ), Hematocrit (Hct), Mean corpuscular volume (MCV), Mean corpuscular haemoglobin (MCH), Mean corpuscular haemoglobin concentration (MCHC), Aspartate aminotransferase (AST), Alanine aminotransferase (ALT), Total protein (TP), Total lipid (TL), Albumin (Alb), Glucose (GLU), Urea and Creatinine (Creat), Lipid peroxidation in kidney LPO(K), Lipid peroxidation in Liver LPO(L), Lipid peroxidation in Gills LPO (G). 
Table 3: The basic data $(\mathrm{N}=4)$ of blood constituent parameters of $C$. gariepinus exposed to lead $(\mathrm{Pb})$, Vitamin E (VE), Lycopene (LYC) and their combinations for 30 day.

\begin{tabular}{|c|c|c|c|c|}
\hline Parameters & Control & LYC & VE & $\mathrm{LYC}+\mathrm{VE}$ \\
\hline Treatments & $\begin{array}{l}\text { Mean } \pm \text { SE } \\
(\text { Min-Max) }\end{array}$ & $\begin{array}{l}\text { Mean } \pm \text { SE } \\
(\text { Min-Max) }\end{array}$ & $\begin{array}{l}\text { Mean } \pm \text { SE } \\
(\text { Min-Max) }\end{array}$ & $\begin{array}{l}\text { Mean } \pm \text { SE } \\
(\text { Min-Max) }\end{array}$ \\
\hline $\mathrm{HB}(\mathrm{g} / 100 \mathrm{ml})$ & $\begin{array}{c}18.28 \pm .38^{\mathrm{C}} \\
(17.59-18.98)\end{array}$ & $\begin{array}{c}19.47 \pm .07^{\mathrm{D}} \\
(19.32-19.62)\end{array}$ & $\begin{array}{c}18.53 \pm .1^{\mathrm{C}} \\
(18.32-18.74)\end{array}$ & $\begin{array}{c}18.56 \pm .18^{\mathrm{C}} \\
(18.22-18.91)\end{array}$ \\
\hline $\begin{array}{c}\text { RBC x } \\
\left(10^{6} / \mathrm{mm}^{3}\right)\end{array}$ & $\begin{array}{c}2.57 \pm .02^{C} \\
(2.51-2.64)\end{array}$ & $\begin{array}{c}2.71 \pm .006^{\mathrm{D}} \\
(2.7-2.73)\end{array}$ & $\begin{array}{l}2.57 \pm .007^{\mathrm{C}} \\
(2.56-2.59)\end{array}$ & $\begin{array}{c}2.6 \pm .01^{\mathrm{C}} \\
(2.56-2.64)\end{array}$ \\
\hline $\operatorname{Hct}(\%)$ & $\begin{array}{c}40.57 \pm .63^{C} \\
(39.05-41.65)\end{array}$ & $\begin{array}{c}44.76 \pm .05^{\mathrm{D}} \\
(44.64-44.88)\end{array}$ & $\begin{array}{c}40.93 \pm .14^{\mathrm{C}} \\
(40.59-41.28)\end{array}$ & $\begin{array}{c}41.79 \pm .41^{\mathrm{C}} \\
(40.79-42.8)\end{array}$ \\
\hline $\operatorname{MCV}\left(\mu \mathrm{m}^{3} /\right.$ cell $)$ & $\begin{array}{c}157.56 \pm 1.82^{\mathrm{C}} \\
(154.47-162.69)\end{array}$ & $\begin{array}{c}165.01 \pm .44^{D} \\
(163.69-165.6)\end{array}$ & $\begin{array}{c}159.27 \pm .25^{\mathrm{CD}} \\
(158.55-159.72)\end{array}$ & $\begin{array}{c}160.75 \pm 1.52^{\mathrm{CD}} \\
(157.53-164.61)\end{array}$ \\
\hline $\mathrm{MCH}(\mu \mathrm{g} /$ cell $)$ & $\begin{array}{c}71 \pm 1.24^{\mathrm{A}} \\
(68.1-73.94)\end{array}$ & $\begin{array}{c}71.77 \pm .32^{\mathrm{A}} \\
(70.95-72.39)\end{array}$ & $\begin{array}{c}72.09 \pm .26^{\mathrm{A}} \\
(71.56-72.72)\end{array}$ & $\begin{array}{c}71.4 \pm .81^{\mathrm{A}} \\
(69.2-72.73)\end{array}$ \\
\hline $\mathrm{MCHC}(\%)$ & $\begin{array}{l}45.05 \pm .34^{\mathrm{AB}} \\
(44.08-45.63)\end{array}$ & $\begin{array}{c}43.49 \pm .1^{\mathrm{A}} \\
(43.27-43.71)\end{array}$ & $\begin{array}{l}44.49 \pm .73^{A} \\
(42.32-45.6)\end{array}$ & $\begin{array}{l}44.41 \pm .22^{A} \\
(43.92-44.9)\end{array}$ \\
\hline AST & $\begin{array}{l}63.98 \pm 3.47^{\mathrm{A}} \\
(53.65-68.59)\end{array}$ & $\begin{array}{l}88.62 \pm 1.21^{\mathrm{B}} \\
(86.22-91.87)\end{array}$ & $\begin{array}{c}65.73 \pm .73^{A} \\
(64.12-67.55)\end{array}$ & $\begin{array}{l}76.51 \pm .48^{\mathrm{AB}} \\
(75.32-77.65)\end{array}$ \\
\hline ALT & $\begin{array}{l}26.16 \pm 1.21^{C} \\
(22.94-28.32)\end{array}$ & $\begin{array}{c}14.11 \pm .27^{\mathrm{A}} \\
(13.51-14.82)\end{array}$ & $\begin{array}{c}16.11 \pm .17^{\mathrm{AB}} \\
(15.77-16.43)\end{array}$ & $\begin{array}{c}19.25 \pm .84^{\mathrm{B}} \\
(17.99-21.68)\end{array}$ \\
\hline $\mathrm{TP}$ & $\begin{array}{c}5.14 \pm .01^{\mathrm{CD}} \\
(5.1-5.19)\end{array}$ & $\begin{array}{l}6.55 \pm .13^{\mathrm{E}} \\
(6.18-6.77)\end{array}$ & $\begin{array}{l}6.34 \pm .008^{\mathrm{E}} \\
(6.33-6.37)\end{array}$ & $\begin{array}{l}6.05 \pm .05^{\mathrm{E}} \\
(5.92-6.16)\end{array}$ \\
\hline TL & $\begin{array}{l}10.79 \pm .22^{\mathrm{DE}} \\
(10.22-11.18)\end{array}$ & $\begin{array}{l}19.37 \pm .29^{\mathrm{G}} \\
(18.86-20.23)\end{array}$ & $\begin{array}{l}15.74 \pm .08^{\mathrm{F}} \\
(15.5-15.89)\end{array}$ & $\begin{array}{l}11.53 \pm .03^{\mathrm{E}} \\
(11.45-11.62)\end{array}$ \\
\hline ALB & $\begin{array}{l}1.77 \pm .04^{\mathrm{C}} \\
(1.69-1.89)\end{array}$ & $\begin{array}{l}2.22 \pm .05^{\mathrm{D}} \\
(2.12-2.37)\end{array}$ & $\begin{array}{l}1.74 \pm .08^{\mathrm{C}} \\
(1.55-1.92)\end{array}$ & $\begin{array}{l}2.25 \pm .09^{\mathrm{D}} \\
(1.99-2.41)\end{array}$ \\
\hline GLU & $\begin{array}{c}62.4 \pm 1.74^{\mathrm{C}} \\
(59.93-67.42)\end{array}$ & $\begin{array}{c}51.06 \pm .9^{\mathrm{B}} \\
(49.04-53.28)\end{array}$ & $\begin{array}{c}34.16 \pm 1.42^{\mathrm{A}} \\
(31.6-38.2)\end{array}$ & $\begin{array}{l}45.22 \pm .9^{\mathrm{B}} \\
(43.1-47.15)\end{array}$ \\
\hline Urea & $\begin{array}{c}12.61 \pm .14^{\mathrm{A}} \\
(12.27-12.96)\end{array}$ & $\begin{array}{l}16.69 \pm .22^{\mathrm{B}} \\
(16.2-17.18)\end{array}$ & $\begin{array}{c}18.68 \pm .2^{\mathrm{B}} \\
(18.17-19.15)\end{array}$ & $\begin{array}{c}10.57 \pm .12^{A} \\
(10.28-10.87)\end{array}$ \\
\hline CREAT & $\begin{array}{r}.46 \pm .007^{\mathrm{A}} \\
(.45-.48)\end{array}$ & $\begin{array}{r}.46 \pm .01^{\mathrm{A}} \\
(.43-.49)\end{array}$ & $\begin{array}{l}.36 \pm .01^{\mathrm{A}} \\
(.33-.39)\end{array}$ & $\begin{array}{l}.4 \pm .01^{\mathrm{A}} \\
(.38-.43)\end{array}$ \\
\hline $\mathrm{LPO}(\mathrm{K})$ & $\begin{array}{l}5.24 \pm .02^{\mathrm{D}} \\
(5.19-5.29)\end{array}$ & $\begin{array}{l}2.81 \pm .01^{\mathrm{C}} \\
(2.78-2.84)\end{array}$ & $\begin{array}{l}2.21 \pm .02^{\mathrm{B}} \\
(2.15-2.27)\end{array}$ & $\begin{array}{c}1.19 \pm .02^{\mathrm{A}} \\
(1.15-1.23)\end{array}$ \\
\hline LPO(L) & $\begin{array}{l}3.83 \pm .02^{\mathrm{D}} \\
(3.79-3.89) \\
\end{array}$ & $\begin{array}{c}1.42 \pm .1^{\mathrm{B}} \\
(1.24-1.67) \\
\end{array}$ & $\begin{array}{l}2.51 \pm .07^{\mathrm{C}} \\
(2.33-2.71) \\
\end{array}$ & $\begin{array}{l}1.13 \pm .01^{\mathrm{A}} \\
(1.1-1.17)\end{array}$ \\
\hline $\mathrm{LPO}(\mathrm{G})$ & $\begin{array}{l}3.16 \pm .02^{\mathrm{D}} \\
(3.12-3.21)\end{array}$ & $\begin{array}{l}1.52 \pm .02^{\mathrm{B}} \\
(1.47-1.58)\end{array}$ & $\begin{array}{l}2.44 \pm .02^{\mathrm{C}} \\
(2.39-2.49)\end{array}$ & $\begin{array}{l}1.23 \pm .01^{\mathrm{A}} \\
(1.19-1.26)\end{array}$ \\
\hline
\end{tabular}

Different letters indicate significance at $\mathrm{p}<0.05$ Red blood cells (RBCs), Haemoglobin $(\mathrm{Hb})$, Hematocrit (Hct), Mean corpuscular volume (MCV), Mean corpuscular haemoglobin (MCH), Mean corpuscular haemoglobin concentration (MCHC), Aspartate aminotransferase (AST), Alanine aminotransferase (ALT), Total protein (TP), Total lipid (TL), Albumin (Alb), Glucose (GLU), Urea and Creatinine (Creat), Lipid peroxidation in kidney LPO(K), Lipid peroxidation in Liver LPO(L), Lipid peroxidation in Gills LPO $(\mathrm{G})$. 
Table 3: Cont

\begin{tabular}{|c|c|c|c|c|}
\hline Parameters & $\mathrm{Pb}$ & $\mathrm{Pb}+\mathrm{LYC}$ & $\mathrm{Pb}+\mathrm{VE}$ & $\mathrm{Pb}+\mathrm{LYC}+\mathrm{VE}$ \\
\hline Treatments & $\begin{array}{l}\text { Mean } \pm \text { SE } \\
(\text { Min-Max) }\end{array}$ & $\begin{array}{l}\text { Mean } \pm \text { SE } \\
\text { (Min-Max) }\end{array}$ & $\begin{array}{l}\text { Mean } \pm \text { SE } \\
\text { (Min-Max) }\end{array}$ & $\begin{array}{l}\text { Mean } \pm \text { SE } \\
\text { (Min-Max) }\end{array}$ \\
\hline $\mathrm{HB}(\mathrm{g} / 100 \mathrm{ml})$ & $\begin{array}{c}14.39 \pm .06^{\mathrm{A}} \\
(14.26-14.53)\end{array}$ & $\begin{array}{l}17.17 \pm .09^{\mathrm{B}} \\
(16.98-17.37)\end{array}$ & $\begin{array}{c}16.85 \pm .1^{\mathrm{B}} \\
(16.65-17.05)\end{array}$ & $\begin{array}{c}17.28 \pm .05^{\mathrm{B}} \\
(17.17-17.42)\end{array}$ \\
\hline $\begin{array}{c}\text { RBC x } \\
\left(10^{6} / \mathrm{mm}^{3}\right)\end{array}$ & $\begin{array}{l}2.06 \pm .03^{\mathrm{A}} \\
(2-2.15)\end{array}$ & $\begin{array}{l}2.45 \pm .02^{\mathrm{B}} \\
(2.42-2.51)\end{array}$ & $\begin{array}{l}2.41 \pm .01^{\mathrm{B}} \\
(2.38-2.45)\end{array}$ & $\begin{array}{l}2.42 \pm .007^{\mathrm{B}} \\
(2.41-2.44)\end{array}$ \\
\hline Hct (\%) & $\begin{array}{c}26.66 \pm .17^{\mathrm{A}} \\
(26.24-27.09)\end{array}$ & $\begin{array}{c}36.79 \pm .3^{\mathrm{B}} \\
(36.18-37.4)\end{array}$ & $\begin{array}{c}36.36 \pm .15^{\mathrm{B}} \\
(36.02-36.71)\end{array}$ & $\begin{array}{c}36 \pm .23^{\mathrm{B}} \\
(35.44-36.56)\end{array}$ \\
\hline $\begin{array}{c}\mathrm{MCV} \\
\left(\mu \mathrm{m}^{3} / \text { cell }\right)\end{array}$ & $\begin{array}{c}129.39 \pm 2.13^{\mathrm{A}} \\
(123.67-133.7)\end{array}$ & $\begin{array}{c}150.01 \pm 1.04^{\mathrm{B}} \\
(148.48-153.08)\end{array}$ & $\begin{array}{c}150.58 \pm .69^{\mathrm{B}} \\
(149.05-152.12)\end{array}$ & $\begin{array}{c}148.6 \pm 1.11^{\mathrm{B}} \\
(146.88-151.7)\end{array}$ \\
\hline $\mathrm{MCH}(\mu \mathrm{g} /$ cell $)$ & $\begin{array}{c}70.26 \pm .67^{\mathrm{A}} \\
(69.02-71.55)\end{array}$ & $\begin{array}{l}70.03 \pm .45^{\mathrm{A}} \\
(69.2-71.27)\end{array}$ & $\begin{array}{c}69.77 \pm .43^{\mathrm{A}} \\
(68.72-70.83)\end{array}$ & $\begin{array}{l}71.34 \pm .16^{\mathrm{A}} \\
(70.98-71.78)\end{array}$ \\
\hline $\mathrm{MCHC}(\%)$ & $\begin{array}{c}53.98 \pm .24^{\mathrm{E}} \\
(53.51-54.45)\end{array}$ & $\begin{array}{l}46.68 \pm .11^{\mathrm{CD}} \\
(46.44-46.93)\end{array}$ & $\begin{array}{l}46.33 \pm .1 \mathrm{BC} \\
(46.1-46.56)\end{array}$ & $\begin{array}{l}48.01 \pm .3^{\mathrm{D}} \\
(47.31-48.6)\end{array}$ \\
\hline AST & $\begin{array}{c}214.79 \pm 6.94^{\mathrm{F}} \\
(195.2-227.98)\end{array}$ & $\begin{array}{c}146.75 \pm 4.65^{\mathrm{E}} \\
(134.12-154.31)\end{array}$ & $\begin{array}{c}107.67 \pm .68^{\mathrm{C}} \\
(106.14-109.12)\end{array}$ & $\begin{array}{c}124.32 \pm 1.71^{\mathrm{D}} \\
(120.39-128.29)\end{array}$ \\
\hline ALT & $\begin{array}{c}81.81 \pm .99^{\mathrm{E}} \\
(79.55-84.34)\end{array}$ & $\begin{array}{l}29.81 \pm 1.86^{\mathrm{C}} \\
(26.25-35.07)\end{array}$ & $\begin{array}{c}35.85 \pm .44^{\mathrm{D}} \\
(34.91-36.98)\end{array}$ & $\begin{array}{c}28.02 \pm .21^{\mathrm{C}} \\
(27.65-28.44)\end{array}$ \\
\hline $\mathrm{TP}$ & $\begin{array}{l}3.11 \pm .01^{\mathrm{A}} \\
(3.08-3.14)\end{array}$ & $\begin{array}{c}5.3 \pm .14^{\mathrm{D}} \\
(4.96-5.63)\end{array}$ & $\begin{array}{l}4.74 \pm .21^{\mathrm{BC}} \\
(4.13-5.11)\end{array}$ & $\begin{array}{l}4.37 \pm .12^{\mathrm{B}} \\
(4.02-4.54)\end{array}$ \\
\hline TL & $\begin{array}{c}4.27 \pm .2^{\mathrm{A}} \\
(3.82-4.74)\end{array}$ & $\begin{array}{l}9.99 \pm .06^{\mathrm{CD}} \\
(9.85-10.11)\end{array}$ & $\begin{array}{c}7.51 \pm .01^{B} \\
(7.48-7.54)\end{array}$ & $\begin{array}{l}9.43 \pm .22^{\mathrm{C}} \\
(8.87-9.89)\end{array}$ \\
\hline ALB & $\begin{array}{l}.75 \pm .01^{\mathrm{A}} \\
(.73-.78)\end{array}$ & $\begin{array}{l}1.03 \pm .004^{\mathrm{B}} \\
(1.02-1.04)\end{array}$ & $\begin{array}{l}1.18 \pm .009^{\mathrm{B}} \\
(1.17-1.21)\end{array}$ & $\begin{array}{c}1.55 \pm .03^{\mathrm{C}} \\
(1.46-1.63)\end{array}$ \\
\hline$\overline{\text { GLU }}$ & $\begin{array}{c}122.09 \pm 2.23^{\mathrm{F}} \\
(117.48-127.45)\end{array}$ & $\begin{array}{l}72.31 \pm 1.45^{\mathrm{D}} \\
(68.99-75.88)\end{array}$ & $\begin{array}{c}103.36 \pm 1.93^{\mathrm{E}} \\
(99.93-107)\end{array}$ & $\begin{array}{c}96.33 \pm 2.85^{\mathrm{E}} \\
(88.32-101.66)\end{array}$ \\
\hline Urea & $\begin{array}{c}43.84 \pm .79^{\mathrm{E}} \\
(41.92-45.77)\end{array}$ & $\begin{array}{c}42.26 \pm .86^{\mathrm{E}} \\
(40.13-44.37)\end{array}$ & $\begin{array}{c}34.47 \pm 1.1^{\mathrm{D}} \\
(32.11-37.17)\end{array}$ & $\begin{array}{l}23.12 \pm 1.57^{\mathrm{C}} \\
(19.16-26.72)\end{array}$ \\
\hline CREAT & $\begin{array}{c}1.08 \pm .07^{\mathrm{C}} \\
(.96-1.3)\end{array}$ & $\begin{array}{l}1.09 \pm .03^{\mathrm{C}} \\
(.99-1.16)\end{array}$ & $\begin{array}{l}.92 \pm .02^{\mathrm{B}} \\
(.86-.99)\end{array}$ & $\begin{array}{l}.76 \pm .02^{\mathrm{B}} \\
(.71-.82)\end{array}$ \\
\hline $\mathrm{LPO}(\mathrm{K})$ & $\begin{array}{c}26.54 \pm .23^{\mathrm{H}} \\
(26.12-27.05)\end{array}$ & $\begin{array}{l}9.53 \pm .01^{\mathrm{F}} \\
(9.49-9.57)\end{array}$ & $\begin{array}{c}12.54 \pm .02^{\mathrm{G}} \\
(12.48-12.58)\end{array}$ & $\begin{array}{c}6.39 \pm .03^{\mathrm{E}} \\
(6.32-6.46)\end{array}$ \\
\hline LPO(L) & $\begin{array}{c}25.22 \pm .03^{\mathrm{H}} \\
(25.15-25.29))\end{array}$ & $\begin{array}{c}8.17 \pm .02^{\mathrm{F}} \\
(8.13-8.23)\end{array}$ & $\begin{array}{c}11.29 \pm .07^{\mathrm{G}} \\
(11.14-11.45)\end{array}$ & $\begin{array}{c}4.97 \pm .05^{\mathrm{E}} \\
(4.85-5.13)\end{array}$ \\
\hline $\mathrm{LPO}(\mathrm{G})$ & $\begin{array}{c}23.53 \pm .02^{\mathrm{H}} \\
(23.57-23.59)\end{array}$ & $\begin{array}{c}6.46 \pm .01^{\mathrm{F}} \\
(6.42-6.51)\end{array}$ & $\begin{array}{l}9.46 \pm .02^{\mathrm{G}} \\
(9.42-9.53)\end{array}$ & $\begin{array}{l}3.35 \pm .009^{\mathrm{E}} \\
(3.33-3.37)\end{array}$ \\
\hline
\end{tabular}

Different letters indicate significance at $\mathrm{p}<0.05$ Red blood cells (RBCs), Haemoglobin $(\mathrm{Hb})$, Hematocrit (Hct), Mean corpuscular volume (MCV), Mean corpuscular haemoglobin (MCH), Mean corpuscular haemoglobin concentration (MCHC), Aspartate aminotransferase (AST), Alanine aminotransferase (ALT), Total protein (TP),Total lipid (TL), Albumin (Alb), Glucose (GLU), Urea and Creatinine (Creat), Lipid peroxidation in kidney LPO(K), Lipid peroxidation in Liver LPO(L), Lipid peroxidation in Gills LPO (G).

The time of exposure main effect was not significant and a positive correlations were recorded between similar $\mathrm{MCV}$ and $\mathrm{MCH}$ in the two periods $(\mathrm{r}$ MCV $=0.926, \quad \mathrm{rMCH}=0.647$

and $\mathrm{rMCHC}=$ 0.930). Diet supplementation with tomato paste and/or vitamin E improved haematological parameters in comparison with the control. 


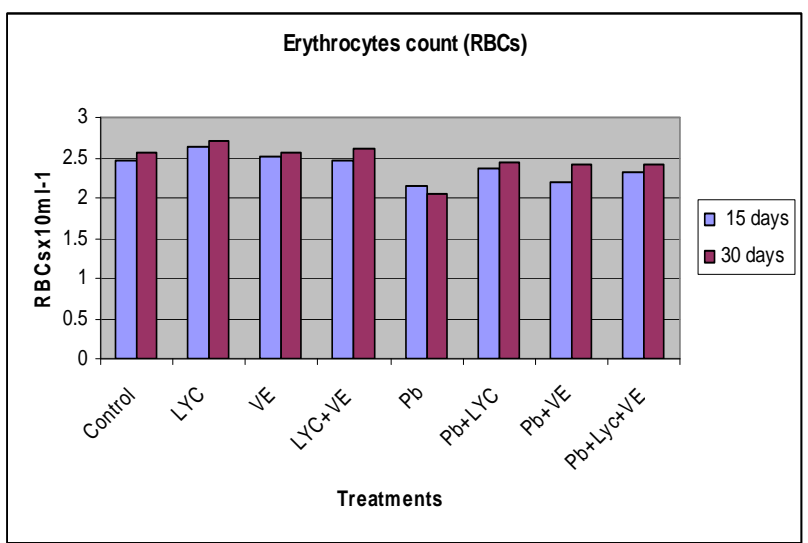

Fig.1: The variability of erythrocytes count $\mathrm{x}$ $\left(10^{6} / \mathrm{mm}^{3}\right)$ in different treatments for 15 and 30 days.

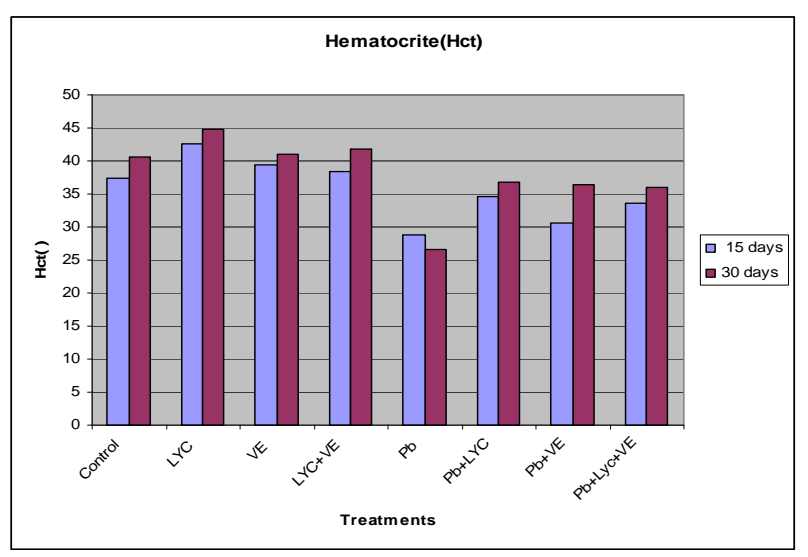

Fig. 3: The variability of haematocrit value (\%) in different treatments for 15 and 30 days.

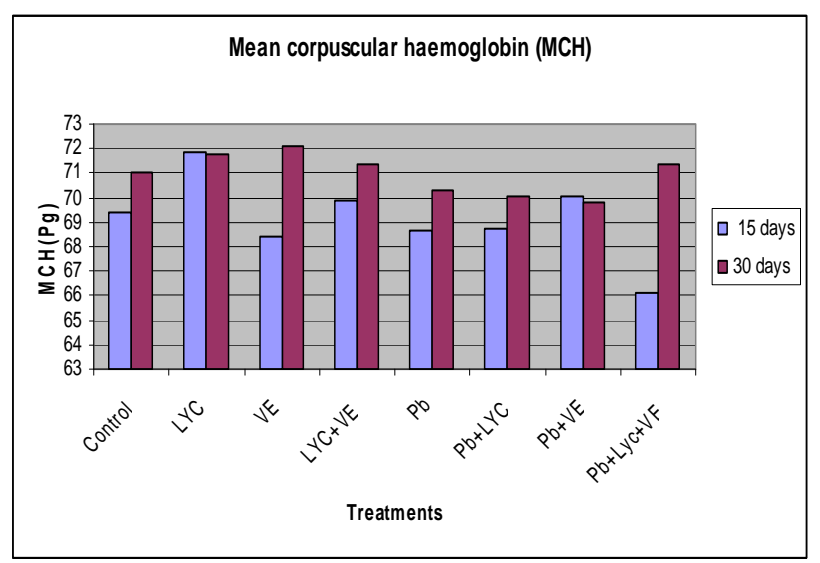

Fig. 5: The variability of mean corpuscular haemoglobin $(\mathrm{MCH})(\mu \mathrm{g} / \mathrm{cell})$ in different treatments for 15 and 30 days.

\section{Biochemical parameters Glucose level}

The normal glucose level of Clarias gariepinus for 15 and 30 days periods are given in Tables $(2 \& 3)$ and Fig. 7. Lead main effect was significantly increased in

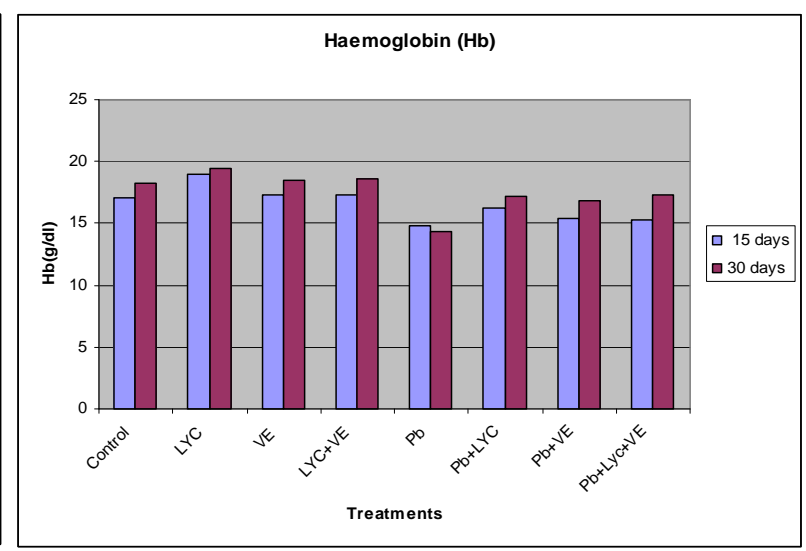

Fig. 2: The variability of haemoglobin content $(\mathrm{g} / 100 \mathrm{ml})$ in different treatments for 15 and 30 days.

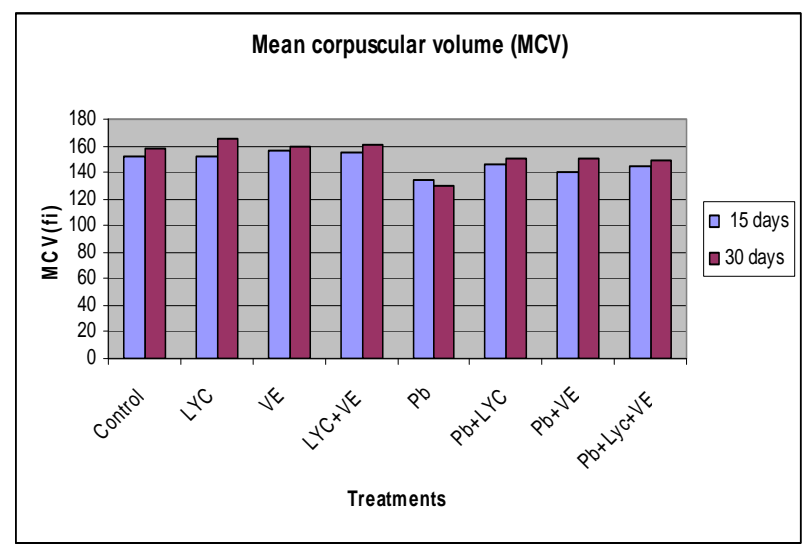

Fig. 4: The variability of mean corpuscular volume (MCV) $\left(\mu \mathrm{m}^{3} /\right.$ cell) in different treatments for 15 and 30 days

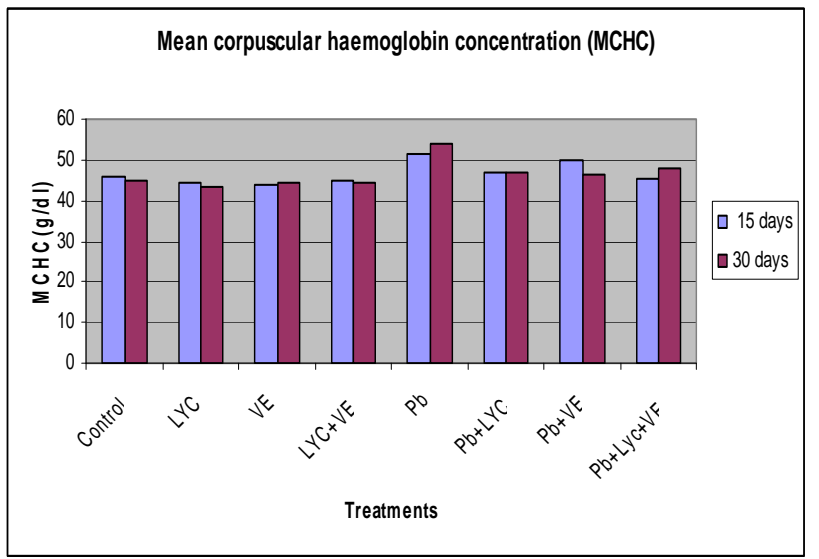

Fig. 6: The variability of mean corpuscular haemoglobin concentration $(\mathrm{MCHC})(\mathrm{g} / \mathrm{dl})$ in different treatments for 15 and 30 days.

both periods $(\mathrm{P}<0.0001)$. In addition, the main effects of $\mathrm{Pb}$, Lycopene, $\mathrm{VE}$ and their interactions were significant in the two periods. 


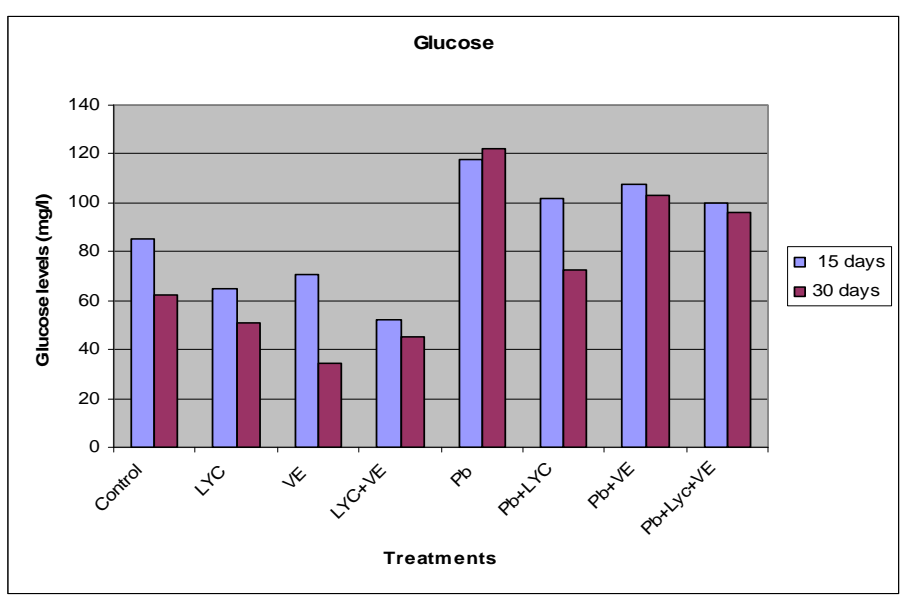

Fig. 7: The variability of glucose $(\mathrm{mg} / \mathrm{L})$ concentrations in different treatments for 15 and 30 days.

\section{Total protein level}

The total protein level of Clarias gariepinus for 15 and 30 days periods are given in Tables ( $2 \& 3)$ and Fig. 8. Lead reflects highly significant decrease in total protein at both periods $(\mathrm{P}<0.0001)$. The main effect of lycopene and /or vitamin $\mathrm{E}$ was significant in the two periods. Pb-LYC-VE main effect was significant at the second period. The time of exposure main effect was not significant and a positive correlation was obtained between the total protein levels in the two periods $(\mathrm{r}$ TP $=.940)$.

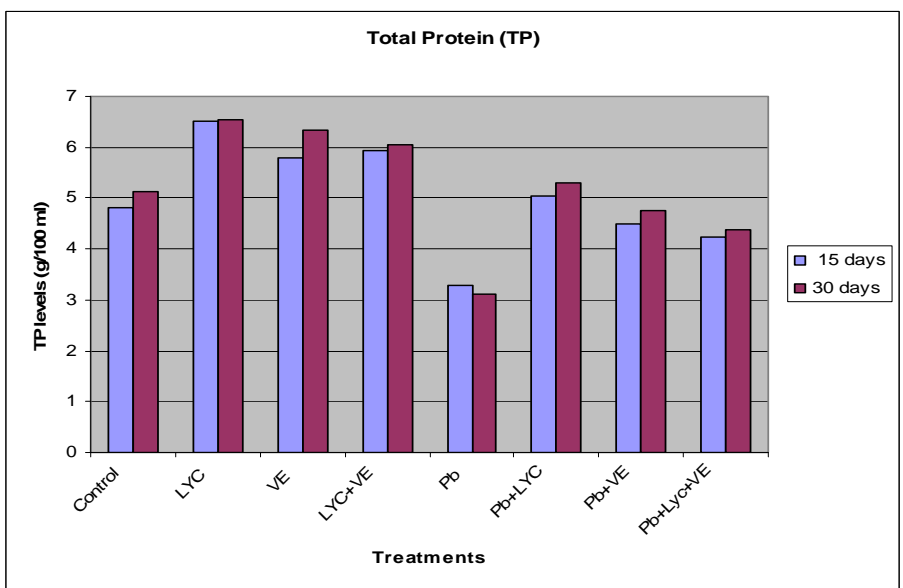

Fig. 8: The variability of total protein $(\mathrm{g} / 100 \mathrm{ml})$ concentrations in different treatments for 15 and 30 days.

\section{Total lipids level}

The total lipids of Clarias gariepinus for 15 and 30 days periods are given in Tables $(2 \& 3)$ and Fig. 9. The lead main effect was highly significant $(\mathrm{P}<0.0001)$ in both exposure periods. Lycopene main effect was highly significant in both exposure periods.
Vitamin E main effect was highly significant in both exposure periods. $\mathrm{Pb}$ LYC-VE main effect was highly significant in both exposure periods. The time of exposure main effect was significant and a positive correlation was obtained between the total lipid levels in the two periods $(\mathrm{r}$ TL $=.995)$. 


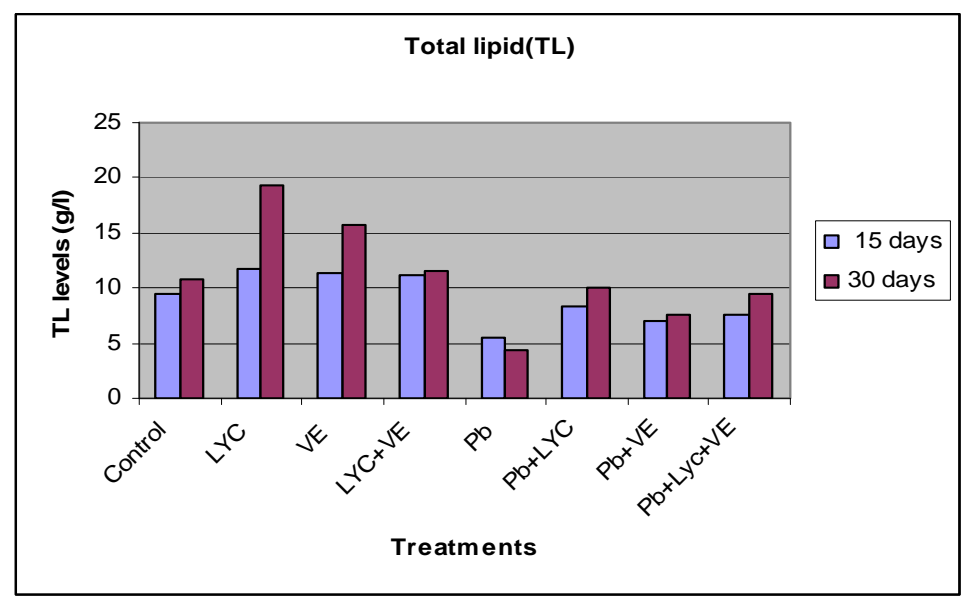

Fig. 9: The variability of total lipids (g/L) concentrations in different treatments for 15 and 30 days.

\section{AST and ALT levels}

The normal values of aspartate amino transferase (AST) and alanine amino transferase (ALT) activity percentage of Clarias gariepinus in the two periods are given in Tables $(2 \& 3)$ and Figs. 10,11. Lead showed highly significant $(\mathrm{P}<0.0001)$ increase in AST and ALT activities in the two periods. The main effect of Lycopene was highly significant in AST and ALT activities in the two periods. No significant main effect of vitamin $E$ was recorded except

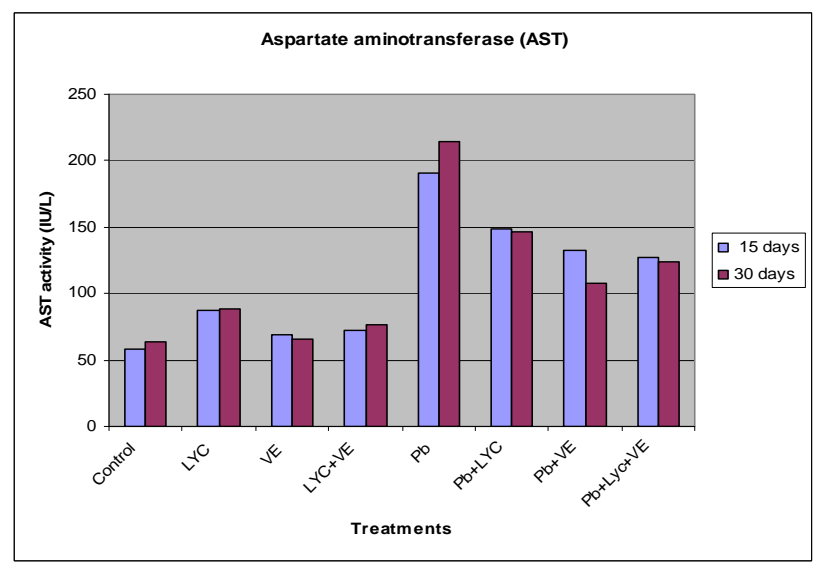

Fig. 10: The variability of aspartate aminotransferase (AST) activity (IU/L) in different treatments for 15 and 30 days.

\section{Albumin level}

The normal values of albumin (Alb) of Clarias gariepinus in the two periods are given in Tables $(2 \& 3)$ and Fig 12. The lead effect was highly for ALT at the second period. Pb-LYCVE interaction showed a significant effect except for ALT at the second period in the two periods. The time of exposure main effect was highly significant for ALT. and a positive correlations were obtained between AST activity at 15 and 30 days in the two periods ( $\mathrm{r}$ AST $=.982$, $\mathrm{rALT}=.986$ ). The diet supplementation with lycopene and /or vitamin $\mathrm{E}$ to $\mathrm{Pb}$-treated fish decreased significantly the activities of AST and ALT serum to the control level.

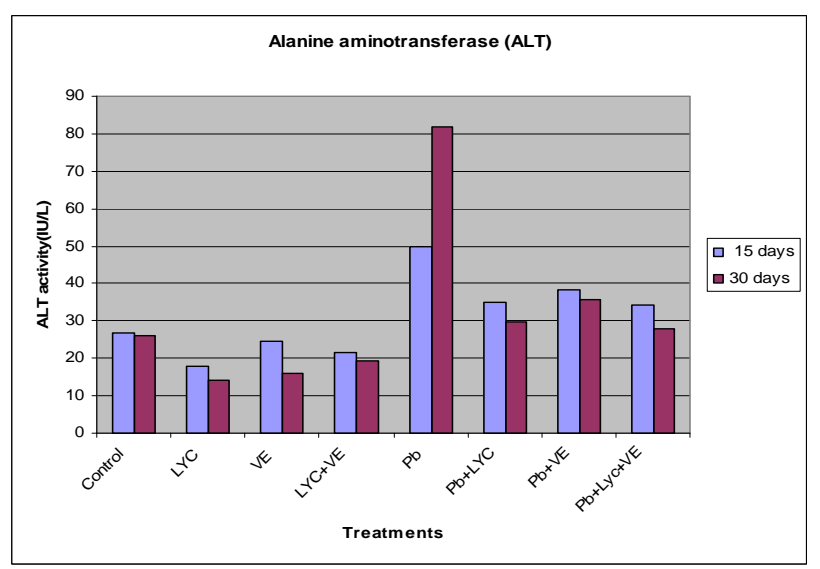

Fig. 11: The variability of alanine aminotransferase (AST) activity (IU/L) in different treatments for 15 and 30 days.

significant $(\mathrm{P}<0.0001)$ decrease in both periods. No significant main effect of vitamin $\mathrm{E}$ was recorded at both periods. Lycopene and LYC-VE interaction was significant only at the second period. $\mathrm{Pb}$ - 
LYC-VE interaction main effect was not significant in both exposure periods. The time of exposure main effect was not significant except $\mathrm{Pb}, \mathrm{LYC}$ and $\mathrm{Pb}-\mathrm{VE}$ time of exposure main effect was significant and a positive correlation was obtained between albumin levels in the two periods ( $\mathrm{ALB}=.914)$.

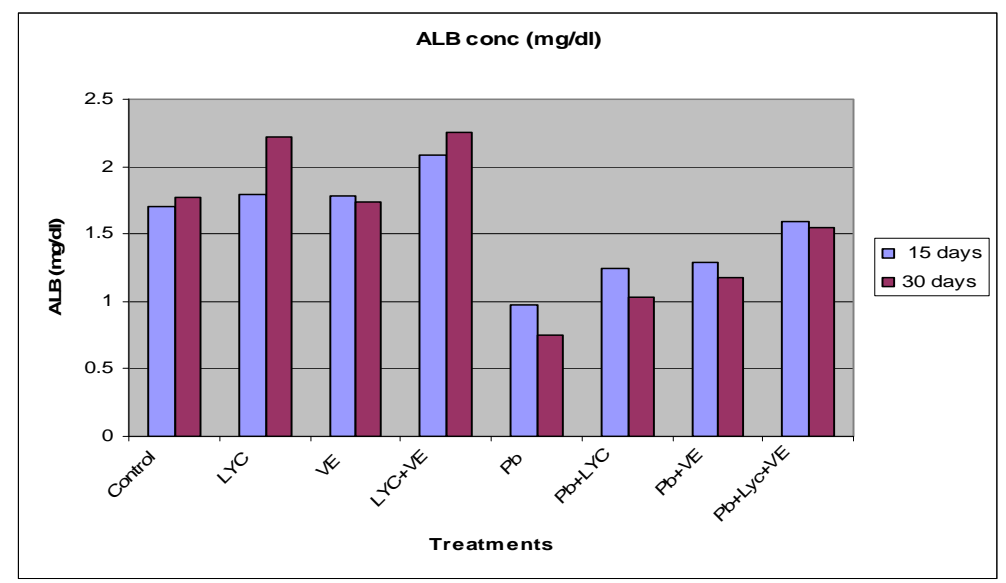

Fig. 12: The variability of Albumin concentrations $(\mathrm{mg} / \mathrm{dl})$ in different treatments for 15 and 30 days.

\section{Urea level}

The normal values of Urea level of Clarias gariepinus in the two periods are given in Tables $(2 \& 3)$ and Fig. 13. The lead effect was highly significant $(\mathrm{P}<0.0001)$ in the two periods .Lycopene and vitamin $\mathrm{E}$ main effects were significant only at the second period. The main effect of $\mathrm{Pb}-\mathrm{LYC}$ was highly significant in both exposure periods $(\mathrm{P}<0.0001)$. The main effect of $\mathrm{Pb}-\mathrm{VE}$ was highly significant in both exposure periods $(\mathrm{P}<0.0001)$. The main effect of $\mathrm{Pb}-\mathrm{LYC}-\mathrm{VE}$ was highly significant in both exposure periods $(\mathrm{P}<0.0001)$ and a positive correlation was obtained between Urea levels in the two periods ( $\mathrm{r}$ urea=.982). The time of exposure main effect was significant except $\mathrm{Pb}-\mathrm{LYC}$-VE time.

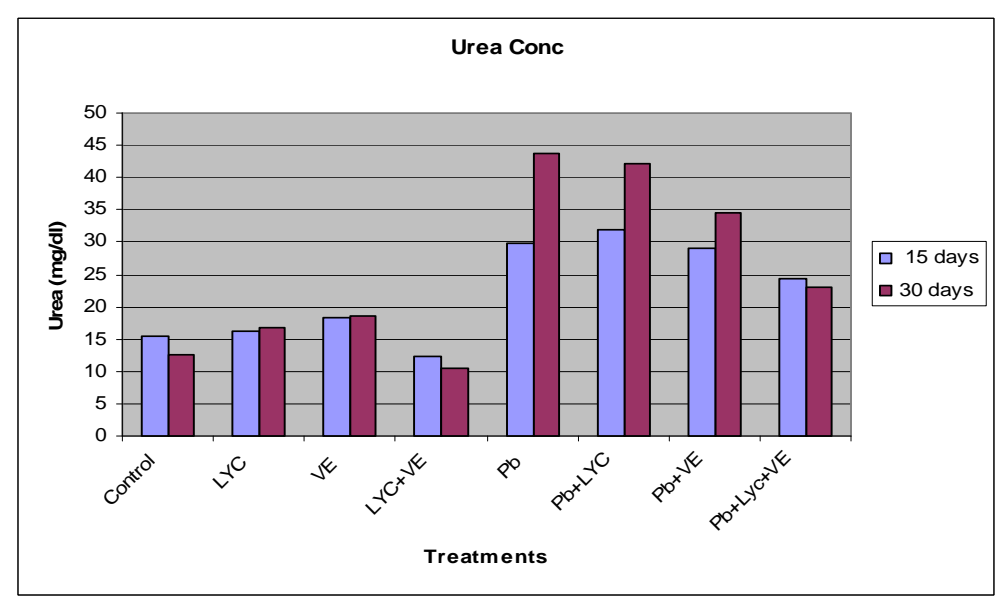

Fig. 13: The variability of Urea concentrations $(\mathrm{Mg} / \mathrm{dl})$ in different treatments for 15 and 30 days.

\section{Creatinine level}

The normal values of Creatinine level of Clarias gariepinus in the two periods are given in Tables $(2 \& 3)$ and Fig. 14. The lead effect was highly significant $(\mathrm{P}<0.0001)$ in the two periods.
Vitamin E main effect was significant only at the first period. Lycopene and LYC-VE interaction was not significant at the two periods. The main effect of $\mathrm{Pb}$ LYC was highly significant in both exposure periods $(\mathrm{P}<0.0001)$. The main 
effect of $\mathrm{Pb}-\mathrm{VE}$ was highly significant in both exposure periods $(\mathrm{P}<0.0001)$. The main effect of $\mathrm{Pb}-\mathrm{LYC}-\mathrm{VE}$ was highly significant in both exposure periods
$(\mathrm{P}<0.0001)$, and a positive correlation was obtained between Creatinine levels in the two periods $(r=.961)$. The time of exposure main effect was not significant.

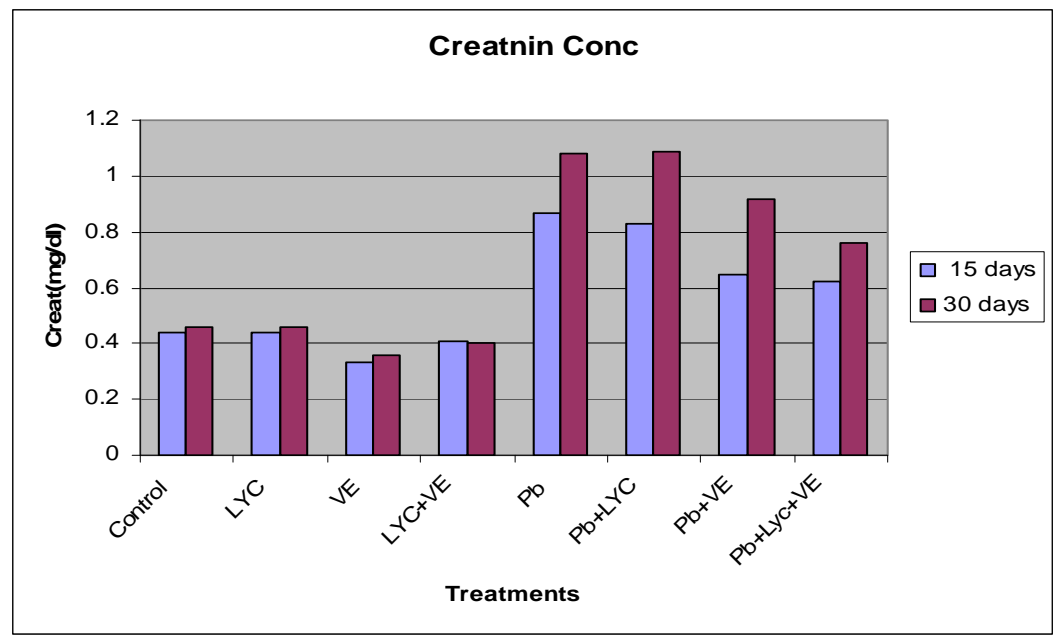

Fig. 14: The variability of Creatinine concentrations $(\mathrm{Mg} / \mathrm{dl})$ in different treatments for 15 and 30 days.

\section{Lipid peroxidation measurement}

The results of lipid peroxidation (LPO) in liver, kidney and gills of Clarias gariepinus in the two periods are given in Tables $(2 \& 3)$ and Figs. 15,16 \&17. The main effects of $\mathrm{Pb}, \mathrm{LYC}, \mathrm{VE}$ and their interactions were highly significant $(\mathrm{P}<0.0001)$ for liver, kidney and gills in the two periods. Similary.
The time of exposure main effect was highly significant $(\mathrm{P}<0.0001)$, $\mathrm{r}(\mathrm{LPO}) \mathrm{L}=1.000, \quad \mathrm{r}(\mathrm{LPO}) \mathrm{k}=1.000$, $\mathrm{r}(\mathrm{LPO}) \mathrm{g}=1.000$. The level of lipid peroxidation (LPO) for liver, kidney and gills was significantly $(\mathrm{P}<0.0001)$ decreased in $\mathrm{Pb}$-exposed fishes fed diets supplemented with Vitamin E and/or Lycopene.

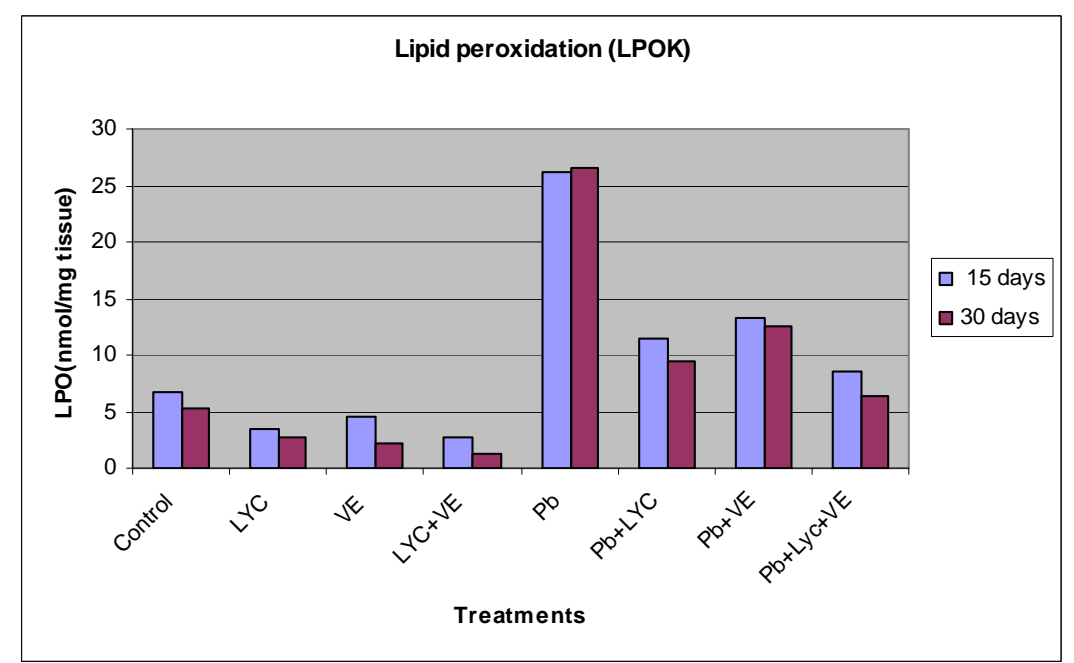

Fig. 15: The variability of lipid peroxidation levels in Kidney (nmol/mg tissue) in different treatments. 


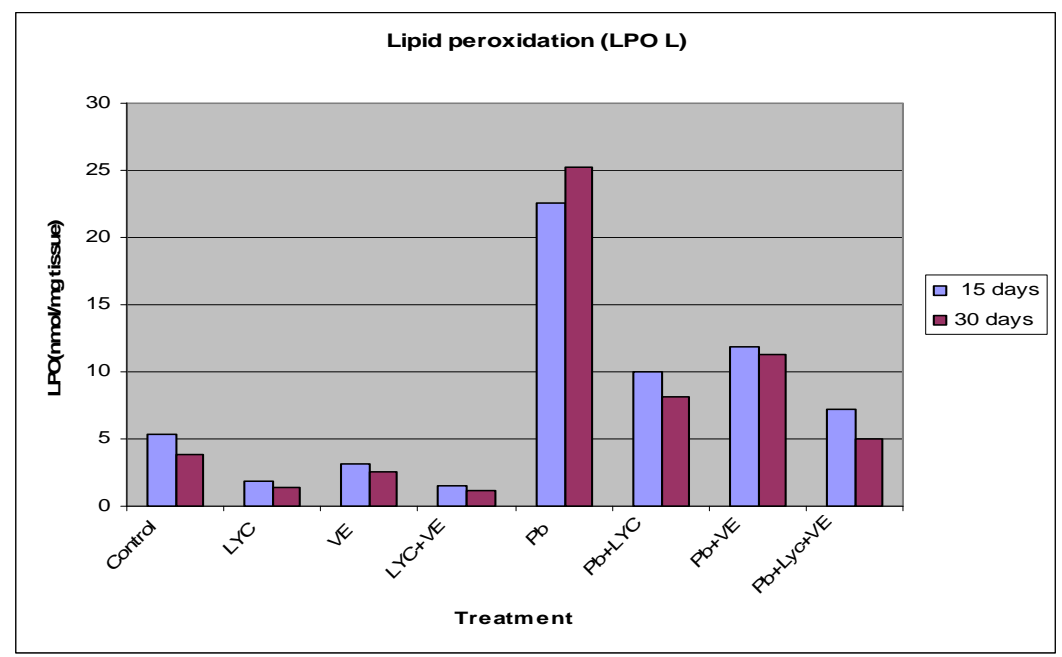

Fig. 16: The variability of lipid peroxidation levels in Liver (nmol/mg tissue) in different treatments

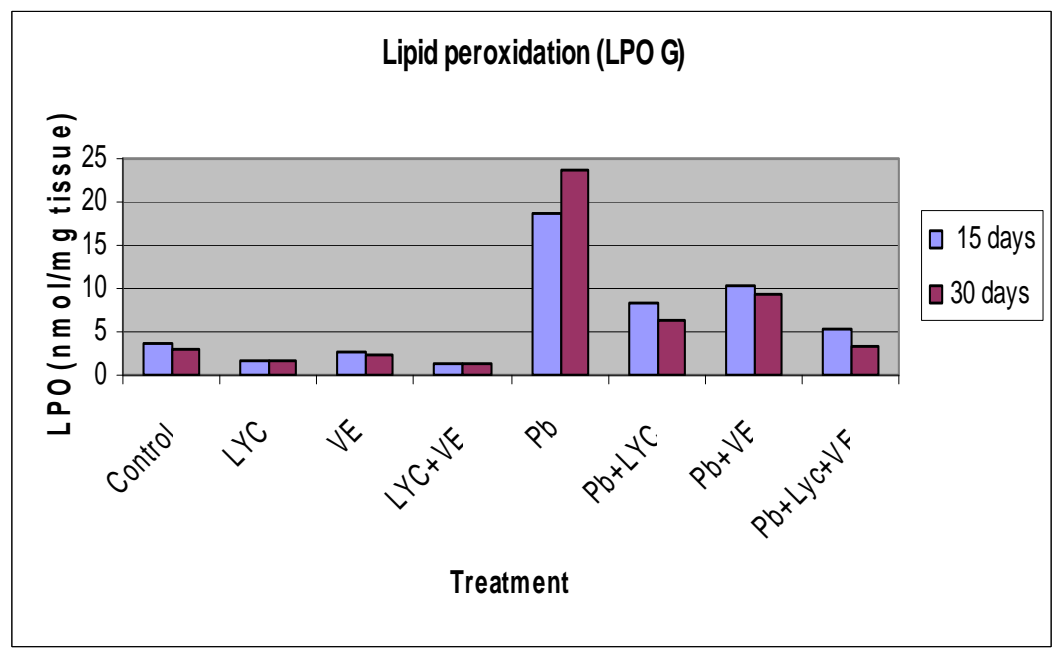

Fig. 17: The variability of lipid peroxidation levels in Gills (nmol/mg tissue) in different treatments.

DISCUSSION

The $\mathrm{Pb}$-exposed fish showed a significant reduction in their $\mathrm{RBCs}, \mathrm{Hb}$ and Hct in comparison with those exposed to $\mathrm{Pb}$ with supplementation of lycopene and vitamin $\mathrm{E}$. These results are in agreement with those of Adeyemo (2007) and Alkahemal -Balawi et al., 2011) who found a significant reduction in $\mathrm{RBCs}$ and $\mathrm{Hb}$ African cat fish (Clarias gariepinus) after exposure to lead. Oluah and Omerebel (2010) found a significant decrease in $\mathrm{Hb}$ value of Clarias gariepinus after exposure to Lead. These alterations were attributed to direct or feedback responses of structural damage to RBC membranes resulting in haemolysis and impairment in haemoglobin synthesis, stress-related release of RBCs from the spleen and hypoxia, induced by exposure to lead (Shah, 2006). These $\mathrm{Pb}$-induced decrease in haematological parameters may be also due disequilibrium of the osmotic pressure inside and outside the blood cell (Heath 1995).

The $\mathrm{Pb}$-induced changes towards increase or decrease in MCV, MCH and $\mathrm{MCHC}$ values were reported in the present work. Similar findings were recorded by Adeyemo (2007) who found asignificant increase in $\mathrm{MCV}, \mathrm{MCH}$ and MCHC values of C. gariepinus after exposure to Lead. Shah, (2006) found asignificant increase in $\mathrm{MCV}, \mathrm{MCH}$ and MCHC values of tench (Tinca tinca) 
after exposure to Lead. Oluah and Omerebel (2010) found decrease in $\mathrm{MCV}, \mathrm{MCH}$ and $\mathrm{MCHC}$ after exposure of Clarias gariepinus to lead. Similar findings were recorded by Ololade and Ogini (2009) after exposure of Clarias gariepinus to Zinc. These chemicalsinduced alterations in $\mathrm{MCV}, \mathrm{MCH}$ and MCHC were attributed to direct or feedback responses of structural damage to $\mathrm{RBC}$ membranes resulting in haemolysis and impairment in haemoglobin synthesis, stress-related release of RBCs from the spleen and hypoxia (Marei et al., 1998; Shah, 2006).

In the present study the increase of serum glucose (hyperglycemia) was revealed in Clarias gariepinus exposed to sublethal concentration of lead. This result is in agreement with that of Alkahemal-Balawi et al. (2011) who found increasing in glucose level (hyperglycemia) of Clarias gariepinus after exposure to lead. Similar findings were observed by Martinez et al., 2004 and Çiftçi et al. (2008) working on Prochilodus lineatus and Anguilla anguilla respectively using lead. Martins et al. (2007) reported a reduced blood glucose level after stress in Atlantic halibut (Hippoglossus hippoglossus L.) fed vitamin E supplemented diets as in the present study.

The present investigation showed decrease in the serum total protein level (hypoproteinemia) after exposure to lead. Similar result was recorded by Martinez et al. (2004) after exposure of Prochilodus lineatus to lead. Çiftçi et al. (2008) reported a decreased total protein level after exposure of Anguilla Anguilla to lead. Such decrease of total protein may be due to destruction of proteinsynthesizing subcellular structures, inhibition of hepatic synthesis of blood protein as a result of heavy metalsprotein interaction or due to stimulated protein catabolism to provide extra energy requirement to overcome the stress in the polluted medium (Fontana et al., 1998).

The present investigation showed decrease in the serum total lipid level. Similar result was recorded by Martinez et al. (2004) in C. gariepinus after exposure to lead. This may be due to the increase in secretion of catechloamines and corticosteroids a result of pollutant stress, which enhanced metabolic rate and in turn reduced metabolic reserves (Fayed et al., 2001).

In the present study,decrease in albumin level was recorded in Pb-treated Clarias gariepinus. The same result was obtained by Mahmoud et al. (2012) by exposure of Clarias gariepinus to mercury. Similar result was obtained by Liao et al.,(1986), who referred to the decline in albumin level, which render the acute turpentine induced inflammation in the presence of no significant change in the concentration of total protein in plasma. Also, they postulated that such situation was accompanied by a corresponding decline in the relative abundance of albumin mRNA in liver. Similar findings were recorded by Mekkawy et al., 1996; Hasheesh et al., 2000; El Fayoumi and Abd Allah (2003), who worked on $O$. niloticus, Chrysichthyes auratus, Cyprinus carpio, and Rainbow trout, respectively. Such results referred to the disturbance in impacts of heavy metals and pesticides on the plasma colloidal osmotic pressure and transportation mechanisms of fatty acids and hormones. Also, the decreased albumin levels (hypoalbuninemia) reflect the active inflammation and serious hepatic and renal damage. The latter damage can not prevent albumin from the blood into urine and being lost. These results may be attributed to liver necrosis (because of toxicant) which, led to leakage from liver into the blood and/or tactual inhibition of liver enzymes.

The current $\mathrm{Pb}$-induced increase in AST and ALT activities can be 
confirmed by similar results reported by Olojo et al. (2012) who found increasing in AST and ALT levels of C. gariepinus after exposure to lead. It has been reported that alterations in enzymes activities in the serum directly indicates major pathologic changes in cell membrane Permeability or hepatic cell rupture (Benjamin, 1978). The diet supplementation with Vitamin E and/ or tomato paste to $\mathrm{Pb}$-treated fish diet led to a significant decreases in the activity of AST and ALT serum. These results are in agreement with those of El-Komy and Hassan (2005) who observed decrease in the activity of AST and ALT of thioacetamide-treated male rat fed tomato-juice as supplemented diet. Kalender et al. (2005) and Ogur et al. (2005) also recorded decrease in activity of AST and ALT after diazinon-induced and nitrate-induced stress in male rats fed vitamin $\mathrm{E}$ as supplemented diets.

In the present study, significant increases in serum urea and creatinine were recorded.These results are confirmed by those of Mahmoud et al. (2012) who reported an increase in the previous parameters in Clarias gariepinus after exposure to mercury. Zaki et al. (2009) found an increase in the urea and creatinine in Oreochromis niloticus due to cadmium exposure. Hadi et al. (2009) reported that the increase of creatinine level might be induced by glomerular insufficiency, increased muscle tissue catabolism or the impairment of carbohydrates metabolism .The diet supplementation with Vitamin $\mathrm{E}$ and /or lycopene to $\mathrm{Pb}$-treated fish decreased levels of serum urea and creatinine to the control level. These results are in agreement with those of Karahan et al. (2005) who observed adecrease in the levels of serum urea and creatinin of gentamicin -treated rats fed tomato juice as supplemented diet.

In the current work, Lead exposure significantly stimulated the levels of oxidized lipids in the form of malondialdehyde (MDA) in the liver, kidney and gills .These results strongly indicated the destructive effect due to an increase of reactive oxygen substances followed by lipid peroxidation. These results are in agreement with those of ElSokkary et al. (2003, 2005) who found asignificant increase in the oxidized lipids in lead- treated animals. The rise in lipid peroxidation level in liver means amodification in the physical characterisitics of cell membrane (Ursini et al., 1991). Since lipid peroxidation leads to hydrolysis of phospholipids into hydroperoxy fatty acids (Salgo et al., 1993). The level of lipid peroxidation was significantly decreased in $\mathrm{Pb}$ exposed fishes fed diets supplemented with vitamin E and lycopene. Similar results for vitamin $\mathrm{E}$ were observed on fish species such as red sea bream, Pagellus bogaraveo (Murata and Yamauchi,1989), channel catfish, Ictalurus punctatus (Gatlin et al.,1992), African catfish ,Clarias gariepinus (Baker and Davies,1996 and 1997), sea bass ,Dicentrarchus labrax (Gatta et al., 2000), Atlantic Salmon, Salmo salar (Scaife et al., 2000) ,juvenile gilthead seabream ,Sparus aurata L. (Mourente et al., 2002), rainbow trout ,Salmo gairdneri (Chaiyapechara et al., 2003), hybrid tilapia, Oreochromis niloticus $\mathrm{x}$ O. aureus (Huang et al., 2003 and 2004), grouper, Epinephelus malabaricus (YuHung and Shi-Yun, 2005) and red hybrid tilapia, Oreochromis sp.(Wang et al., 2006).

Lycopene was found by many authors to improve stress-induced lipid peroxidation in rats (Bhuvaneswaria et al 2001, Velmurugan et al., 2001 and 2002, El-Demerdash et al. 2004, El-Komy and Hassan, 2005, Moreira et al., 2005, Atessahin et al., 2006 and Yilmaz et al ., 2006).

\section{REFERENCES}

Abdallah, G.M.; El-Sayed, S.M. and Abo-Salem, O.M. (2010). Effect of 
lead toxicity on coenzyme Q levels in rat tissues. Food Chem. Toxicol. 48: 1753-1756.

Ademuyiwa, O.; Ugbaja, R. N.; Rotimi, S. O.; Abam, E. and Okediran, B. S. (2007). Erythrocyte acetylcholin esterase activity as asurrogate indicator of lead induced neurotoxicity in occupational lead exposure in Abeokuta, Nigeria. Environ. Toxicol. Pharmacol, 24:183188.

Adeyemo, O.K. (2007). Haematological Profile of Clarias gariepinus (Burchell, 1822) Exposed to Lead. Turk.J. Fish. Aquat. Sci., 7: 163-169.

Alkahem Al-Balawi, H.F.; Ahmad, Z.; Al-Akel, A.S.; Al-Misned, F.; Suliman, E.M. and Al-Ghanim, K. A. (2011). Toxicity bioassay of lead acetate and effects of its sublethal exposure on growth, haematological parameters and reproduction in Clarias gariepinus, African Journal of Biotechnology, 10(53): 11039-11047. Almeida, J. A. ; Diniz, Y. S.; Marques, S. F. G.; Faine, L. A.; Ribas, B. O.; Burneiko, R. C. and Novelli, E. L. B. (2002). The use of oxidative stress responses as biomarkers in Nile tilapia (Oreochromis niloticus) exposed to in vivo cadmium contamination. Environ. Int., 27: 673679.

Anwar, W. A. (2003). Environmental health in Egypt. International Journal of Hygiene and Environmental Health, 206: 339-350.

Atessahin, A.; Karahan, I.; Türk, G.; Gür, S.; Yilmaz, S. and Ceribas, I. A. O. (2006). Protective role of lycopene on cisplatin-induced changes in sperm characteristics, testicular damage and oxidative stress in rats. Reprod. Toxicol., 21: 42-47.

Baker, R.T.M. and Davies, S.J. (1996). Changes in tissue $\alpha$ - tocopherol status and degree of lipid peroxidation with varying $\alpha$-tocopheryl acetate inclusion in diets for African catfish. Aquacult. Nutr., 2: 71-79.

Baker, R.T.M. and Davies, S.J. (1997). The quantitative requirement for $\alpha$ tocopherol by juvenile African catfish, Clarias gariepinus Burchell. Anim. Sci., 65: 135-142.

Basa, S. P.; Usha Rani, A. (2003). Cadmium induced antioxidant defense mechanism in freshwater teleost Oreochromis mossambicus (Tilapia). Eco. Toxicol. Environ. Saf., 56 (2): 218-221.

Benjamin, M. N. (1978). Outline of veterinary clinical pathology. Iowa, USA, University press, 229-232.

Berrahal, A. A.; Nehdi, A.; Hajjaji, N.; Gharbi, N. and El-Fazaa, S. (2007). Antioxidant enzymes activities and bilirubin level in adult rat treated with lead. Comptes. Rendus. Biol. 330: 581-588.

Bhuvaneswaria, V.; Velmurugana, B.; Balasenthila, S; Rama Chandranb, C. R; Naginia, S. (2001). Chemopreventive efficacy of lycopene on 7, 12-dimethylbenz [a] anthracene-induced hamster buccal pouch carcinogenesis. Fitoterapia ,72: 865-874.

Canli, M. (1995). Natural occurrence of metallothionein like proteins in the hepatopancreas of the Norway lobster Nephrops Norvegicus and effects of $\mathrm{Cd}, \mathrm{Cu}$, and $\mathrm{Zn}$ exposures on levels of the metal bound on metallothionein. Turk. J. Zool., 19, 313-321.

Capanoglu E, Beekwilder J, Boyacioglu D, Hall R, de Vos R. (2008). Changes in antioxidant and metabolite profiles during production of tomato paste. $\mathbf{J}$ Agric Food Chem 56:964-973.

Chaiyapechara, S.; Casten, M.T.; Hardy, R.W. and Dong, F.M. (2003). Fish performance, fillet characteristics, and health assessment index of rainbow trout (Oncorhymchus mykiss) fed diets containing adequate and high concentrations of lipid and 
vitamin E. Aquaculture, 219: 715738.

Choudhari, S. M. and Ananthanarayan, L. (2007). Enzyme aided extraction of lycopene from tomato tissues. Food Chem., 102: 77-81.

Ciftci-Soydemir, N; Cicik, B.; Erdem, C. and Ay, O. (2008). Effects of Lead Concentrations on Sera Parameters and Hematocrit Levels in Anguilla anguilla L, 1758. J. Fish Sci. Com., 2(4): 616-622.

Cloes , E.H. (1980). Veterinary clinical pathology. W. Saunder company, London.

Dacie, J.V. and Lewis, S.M. (1984). Practical Haematology. Churchill Living Stone. London.

Denny, P.; Hart, B. T.; Lasheen, B. T.; Supermanian, V. and Wong, M. H. (1987). Lead. In Lead, Mercury, Cadmium and Arsenic in the Environment(ed. Hutchinson, T. C. a. M., K.M., pp. 3-16, John Willy and Sons Ltd. Chechester, Great Britian.

DeWilde, M.A. and Houston, A.H. (1967). Haematological aspects of the thermoacclimatory process in rainbow trout Salmo gairdnerii, J. Fish. Bd. Can., 24:2267-2281.

DiMascio, P.; Kraiser, S. and Sies, H. (1989). Lycopene as the most effective biological carotenoid singlet oxygen quencher. Arch Biochem Biophys, 274:532-538.

El-Demerdash, F.M.; Yousef, M.I.; Kedwany, F. S. and Baghdadi, H. H. (2004). Cadmium-induced changes in lipid peroxidation, blood hematology, biochemical parameters and semen quality of male rats: protective role of vitamin $E$ and b-carotene. Food Chem Toxicol ., 42:1563-1571.

El-Fayoumi, R. I. and Abd-Allah, G. A. (2003). Toxicity of fumonisin B1 in Rainbow trout. . J. Egypt. Ger. Soc. Zool., 40: 489-502.

El-Komy, M.M. and Hassan, H.A. (2005). The role of tomato-juice as a protective agent against thioacetamide hepatotoxicity in male rats. Comp. Physio., 46(A): 217-234.

El-Sokkary, G.H.; Abdel-Rahman, G.H. and Kamel, E.S. (2005): Melatonin protects against lead-induced hepatic and renal toxicity in male rats. Toxicology., 213: 25-33.

El-Sokkary, G. H.; Kamel, S. S. and Reiter, R. J. (2003). Prophylactic effect of melatonin in reducing leadinduced neurotoxicity in the rat. Cell. Mol. Biol. Lett., 8: 461-470.

Emmerson, B. T. (1973). Chronic Lead Nephropathy. Kidney International 4: 1-5.

Falke, H. E. and Xwennis, W. C. M. (1990). Toxicity of lead acetate to female rabbits after chronic subcutaneous administration. 1 . Biochemical and clinical effects. Arch. Toxicol., 64: 322-329.

Farkas, A., Salanki, J.; Specziar, A. (2002). Relation between growth and the heavy metal concentration in organs of bream Abramis brama L. populating lake Balaton. Arch. Environ. Contam. Toxicol., 43 (2): 236-243.

Farrag, A.R.H.; Mahd, K. A.; Abdel Rahman, G. H. and Osfor, M. M. (2007). Protective effect of Nigella Sative seeds against lead induced hepatorenal damage in male rats .Pak. J. Biol. Sci., 10:2809-2816.

Fayed, H. M.; Zaghloul, K. H.; Abd ElMonem, S. and Mohamed, H. A. (2001). Biological responses of the Nile Tilapia, Oreochromis niloticus and the Nile catfish, Clarias gariepinus induced by agricultural and industrial pollutants. J. Union Arab Biol., 16(A): 543-568.

Fontana, L.; Moreira, E.; Torres, M.; Ferna 'ndez, I.; Rios ,A.; Sanchez de Medina ,F. and Gil, A. (1998). Dietary nucleotides correct plasma and liver microsomal fatty acids alterations in rats with liver cirrhosis induced by oral intake of thioacetamide. J Hepatol 28:662-669. 
Frei, B. (1991): Ascorbic acid protects lipids in human plasma and lowdensity lipoprotein against oxidative damage. Amer. J. Clin. Nutr., 24:1113-1118.

Gardner, G. R. and Yevich, P. P. (1969). Toxicological effects of cadmium on Fundulus heteroclitus under various oxygen, $\mathrm{Ph}$, salinity and temprture regims Ame. Zool., 1096-1099.

Gatlin, D. M. and Wilson, R.P. (1986). Dietary copper requirement of fingerling channel catfish. Aquacult., 54: 277-85.

Gatta, P.P.; Pirini, M.; Testi, S.; Vignola, G. and Monetti, P.G. (2000). The influence of different levels of dietary vitamin E on sea bass, Dicentrarchus labrax flesh quality. Aquacult. Nutr., 6: 47-52.

Hadi, A., Shokr, A. and Alwan, S. (2009). Effects of aluminum on the biochemical parameters of fresh waterfish Tilapia zillii, J. Sci. Applica., 3: 33-41.

Handy, R. D. (1994). Intermittent exposure to aquatic pollutants assessment, toxicity and sublethal responses in fish and invertebrates. Comparative Biochemistry and Physiology C-Pharmacology Toxicology \& Endocrinology, 107: 171-184.

Hao, S.; Tian, P.; Tang, W. and Ru, B. (2002). Protective effect of extra metallothionins from rabbit liver induced by zinc on toxicity of lead in rat primary hepatocyte culture. Wei Sheng Yan Jiu ., 31: 229-231.

Harrison, P. R. and Winchest, J. W. (1971). Area wide distribution of lead, copper, and cadmium in air particulates from Chicago and Northwest Indiana. Atmospheric Environment ., 5: 863-873.

Hasheesh, S. W.; Haggag, A. M.; Mohamed-Assem, S. M. and Ashraf, M. (2000). Influence of the herbicide fluometuron on some physiological and biochemical characteristics of the common carp, Cyprinus carpio. J. Union Arab Biol. Cairo 14: 233-256.

Heath, A.G .(1995). Water pollution and fish physiology. CRC Press, Boca Raton., 227:869-880.

Hillam, R. P. and Ozkan, A. N. (1986). Comparison of local and systemic immunity after intratracheal intraperitoneal, and intravenous immunization of mice exposed to either aerosolized or ingested lead. Environ. Res., 39: 265-277.

Honchel, R.; Marsano, L.; Cohen, D.; Shedlofsky, S. and McClain, C. J. (1991). Lead enhances lipopolysaccharide and tumor necrosis factor liver injury. J. Lab. Clin. Med., 117: 202-208.

Huang, C. H.; Chang, R. J.; Huang, S. L. and Chen, W. (2003). Dietary vitamin E supplementation affects tissue lipid peroxidation of hybrid tilapia, Oreochromis niloticus x O. aureus. Comp. Biochem. Physiol. B: Biochem. Mol. Biol., 134(B): 265270.

Huang, S. L.; Weng, Y. M. and Huang, C. H. (2004). Lipid peroxidation in sarcoplasmic reticulum and muscle of tilapia is inhibited by dietary vitamin E supplementation. J. Food Biochem., 28: 101- 111.

Juneja, C. and Mahajan, C. (1983). Hematological and haemopoietic changes in fish Channa punctatus due to mercury pollution in water, Indian J. Anim. Sci., 17: 63-71.

Kalender, S.; Ogutcu, A.; Uzunhisarcikli, M.; Acikgoz, F.;Durak, D.; Ulusoy, Y. and Kalender, Y. (2005). Diazinon-induced hepatotoxicity and protective effect of vitamin $\mathrm{E}$ on some biochemical indices and ultrastructural changes. Toxicology., 211: 197-206.

Karahan, I.; Atessahin, A.; Yilmaz, S.; Ceribasi, A.O. and Sakin, F. (2005). Protective effect of lycopene on gentamicin-induced oxidative sress 
and nephrotoxicity in rats.

Toxicology., 215: 198-204.

Kori-Siakpere, O., Ake, J.E.G. and Avworo, U.M. (2006). Sublethal effects of some selected haematological parameters of Heteroclarias (A hybrid of Heterobranchus bidorsalis and Clarias gariepinus), Int. J. Zool. Res., 2 : 77- 83.

Liao, W. S.; Jefferson, L. S. and Taylor, J. M. (1986). Changes in plasma albumin concentration, synthesis rate , andmRNA level during acute inflammation.Am J Physiol.251(6pt1) :C928-934.

Maheswaran, R.; Devapaul, A.; Muralidharan, S.; Velmurugan, B. and Ignacimuthu, S. (2008). Haematological studies of fresh water fish, Clarias batrachus (L.) exposed to mercuric chloride. Int. J. Integ. Biol., 2: 49-54.

Mahmoud, U. M.; Mekkawy, I. A. A and Ibrahim, A. T. A. (2012). Biochemical Response of the African Catfish, Clarias gariepinus (Burchell, 1822) to Sublethal Concentrations of Mercury Chloride with Supplementation of Selenium and Vitamin E .Toxicol. Environ. Health. Sci., 4(4): 218-234.

Marei, M.A.S.; Haggag, A.M. and ElBadawy, A.A. (1998). Physiological and biochemical responses of the common carp, Cyprinus carpio, to an organophosphorous insecticide "Profenofos". Egypt. J. Zool., 31: 279-302.

Marchlewicz, M.; protasouicki, M.; Rozewicka; Piaseecka, M. and Lasezczynska, M. (1993). Effect of long-term exposure to lead on testis and epididymis in rats. FoliaHistochem. Cytobiol., 31: 55-62.

Martins, D.A.; Afonso, L.O.B.; Hosoya, S.; Lewis-Mc Crea , L.M.; P., V.L.M. and Lall, S.P. (2007). Effects of moderately oxidized dietary lipid and the role of vitamin $\mathrm{E}$ on the stress

response in Atlantic halibut (Hippoglossus hippoglossus L.). Aquaculture, 272(1- 4): 573-580.

Martinez, C. B. R., M. Y. Nagae, C. T. B. V. Zaia and D. A. M. Zaia. (2004). Morphological and physiological acute effects of lead in the neotropical fish Prochilodus lineatus. Brazilian Journal of Biology, 64 (4): 797-807.

Matos, P.; Fonta1'nhas-Fernandes, A.; Peixoto, F.;Carrola, J. and Rocha, E. (2007). Biochemical and histological hepatic changes of Nile tilapia Oreochromis niloticus exposed to carbaryl. Pestic. Biochem. Physiol., 89: 73-80.

Mekkawy, I.A.A.; Hussein, S.Y.; AbdelNasser, M. and Ahmed, S.H.M. (1996). Comparative studies on the effects of herbicide atrazine on some blood constituent and protein electrophoretic patterns of Oreochromis niloticus and Chrysichthyes auratus. J Egypt Ger Soc Zool ., 19(A):283-319.

Moreira, E.A.M.; Fagundes , R.L.M.; Filho, D.W.; Neves , D.; Sell , F.; Bellisle, F. and Kupek, E. (2005). Effects of diet energy level and tomato powder consumption on antioxidant status in rats. Clin Nutr., 24:1038-1046.

Mourente, G.; Diaz, E.; Bell, J. and Tocher, D. (2002). Increased activities of hepatic antioxidant defense enzymes in juvenile gilthead sea bream (Sparus aurata L) fed dietary oxidized oil: attenuation by dietary vitamin E. Aquaculture, 214(1- 4): 343 - 361.

Murata, H. and Yamauchi, K. (1989). Relationship between 2-thiobarbituric acid values of some tissues from cultured red sea bream and its dietary $\alpha$-tocopherol levels. Nippon Suisan Gakkaishi., 55: 1435-1439.

Ogur, R.; Coskun , O.; Korkmaz, A.; Oter, S.; Yaren , H . and Hasde, M. (2005). High nitrate intake impairs 
liver functions and morphology in rats; protective effects of atocopherol.Environ Toxicol Pharmacol., 20:161-166.

Ohkawa, H.; Ohishi, N. and Nagi, K. (1979). Assay for lipid peroxides in animal tissues by thiobarbituric acid reaction. Anal Biochem, 95:351-358.

Okajima, E.; Tsutsumi, M.; Ozono, S.; Akai, H.; Denda, A.; Nishino, H.; Oshima, S.; Sakamoto, H. and Konishi,Y. (1998). Inhibitory effect of tomato juice on rat urinary bladder carcinogenesis after N-Butyl-N-(4hydroxybutyl)nitrosamine Initiation. Jpn J Cancer Res., 89:22-26.

Olojo, E.A.A.; Abass, A. A.; Olurin, K. B. and Mbaka, G. (2012). The potential use of certain protein metabolism parameters as biomarkers of heavy metal (lead) stress in the African cat fish ,Clarias gariepinus. Agricultural J., 7(5):316-322.

Ololade, I.A. and Oginni, O. (2010). Toxic stress and hematological effects of nickel on African catfish, Clarias gariepinus, fingerlings, Environ. Toxicol. Chem., 2: 014019.

Ololade, I. A. and Oginni, O. (2009). Toxic stress and hematological effects of nickel on African catfish, Clarias gariepinus, fingerlings, Environ. Toxicol. Chem., 2: 014019.

Oluah, N. S. and Omerebele, U. A. M. (2010). Changes in haematological parameters of Clarias gariepinus exposed to lead poisoning. J. fisher. Inter., 5(4):72-76.

Ortuno, J.; Cuesta,A.; Esteban, M. A .and Meseguer, J. (2001). Effect of oral administration of high vitamin $\mathrm{C}$ and E dosages on the gilthead seabream (Sparus aurata L.) innate immune system. Vet Immun Immuno., 79:167-180.

Osman, A.G.M.; Koutb, M. and Sayed, A.E.H.(2010). Use of hematological parameters to assess the efficiency of quince (Cydonia oblonga Miller) leaf extract in alleviation of the effect of ultraviolet -A radiation on African catfish Clarias gariepinus (Burchell, 1822), J. Photochem. Photobiol. B: Biol., 99: 1-8.

Park, J.W.; Rinchard, J.; Liu, F.; Anderson, T.A.; Kendall, R. J. and Theodorakis, C.W. (2006). The thyroid endocrine disruptor perchlorate affects reproduction, growth, and survival of mosquitofish, Ecotoxicol. Environ. Saf., 63:343-52.

Patrick, L. (2006). Lead toxicity part II: the role of free radical damage and the use of antioxidants in the pathology and treatment of lead toxicity. Altern Med Rev., 11:114127.

Ranganna, S. (1976). Manual of Analysis of Fruits and Vegetable Products McGraw Hill. New Delhi, 77 pp.

Ranzani-Paiva, M.; Salles, F.; Eiras, J.; Ishikawa, C. and Alexandrino, A. (1999). hematolo gica de curimbat(Prochilodus scrofa), pacu (Piractus mesopotamicus) e tambaqui (Colossoma macropomum) das estaç? Bol. Inst. Pesca Sao Paulo., 25:77-83.

Reichenbach-Klink, H. H. (1966). The blood components of fish with relation to parasiyies infection and water pollution. Bull. Int. Epizool., 65:1039-1054.

Reitman, S. and Frankel, S. (1957): A colorimetric method for the determination of serum glutamic oxalacetic and glutamic pyruvic transaminases. Am J Clin Pathol 28:56-63.

Rodriguez, A.; Cuesta, A.; Esteban, M.A. and Mesegure, J. (2004). The effect of dietary of administration of the fungus Mucro circinelloides on nonspecific immune responses of gilthead seabream. Fish Shellfish Immunol, 16:241-249.

Royce, E. S.; Herdert, L. and Needlleman, E. (1990). Case studies 
in environmental medicine. Lead toxicity. ATSDR: 2-8.

Salgo, M.G.; Corongiu, F.P. and Sevnian, A. (1993). Enhanced interfacial catalysis and hydrolytic specificity of phospholipase A2 toward peroxidized phosphatidylcholine vesicles. Arch. Biochem. Biophys., 304: 123-132.

Sandmann, G. (1994). Carotenoid biosynthesis in microorganisms and plants. Eur. J. Biochem., 223: 7- 24.

Scaife, J.R.; Onibi, G.E.; Murray, I.; Fletcher, T.C. and Houlihan, D.F. (2000). Influence of $\alpha$-tocopherol acetate on the short- and longterm storage properties of fillets from Atlantic salmon Salmo salar fed a high lipid diet. Aquacult. Nutr., 6: 6571.

Shafiq-ur-Rehman, (1991). Effects of lead on the behavioral complex stereotypes and regional brain Dopamine levels in rats. Arch. Environ. Contam. Toxicol., 20: 527530.

Shah, S.L. (2006). Haematological parameters in tench, Tinca tinca after short-term exposure to lead. J. Appl. Toxicol., 26 (3): 223-228.

Shukla, R.; Dietrich, K. N.; Bornchein, R. L.; Berger, O. and Hammond, P. B. (1991). Lead exposure and growth in the early prechool child: A follow up report form the Cincinnati lead study. Pediatrics., 88: 886.

SPSS. (1998). SPSS for Windows, Release 9.0.0 SPSS Inc.

Sies, H. (1997). Oxidative stress: oxidants and antioxidants. Exp. Physiol., 82(2): 291-295.

Srivastava, S. and Choudhary, S.K. (2010). Effect of artificial photoperiod on the blood cell indices of the catfish, Clarias batrachus, Journal of Stress Physiology and Biochemistry., 6: 22-32.

Stoskopf, M.K. (1993). Fish medicine. W. B.Saunders Co, Philadelphia Tort, L. and Torres, P. (1988). The effects of sub lethal concentration of cadmium on hematological parameters in the dog fish, Scyliorhinus Caniccula. J. Fish. Biol., 32 (2): 277-282.

Traber, M.G. and Packer, L. (1995): Vitamin E: Beyond antioxidant function. Amer. J. Clin. Nutr., 62: 15015 - 15095 .

Upasani, C. D.; Khera, A. and Balaraman, R. (2001). Effect of lead with Vitamin E, C. or Sqirulina on malondialdehyde: conjugated dienes and hydroperoxides in rats. Indian J. Exp. Biol., 39: 70-74.

Ursini, F.; Maiorino, M. and Sevanian, A. (1991): Membrane hydroperoxides In: Sies, H. (Ed.), Oxidative stress: Oxidants and Antioxidants. Academic Press Ltd, London, 319336.

U. S. Department of Agriculture, Agriculture Research Service (2004). USDA National Nutrition Database for Standard Reference, Release, 116.

Vankampen EJ (1961). Determination of hemoglobin. Clinica Chimica Acta, 6: 538-544.

Velmurugan, B.; Bhuvaneswari, V. and Nagini, S. (2002). Antiperoxidative effects of lycopene during N-methyl$\mathrm{N}$-nitro-N-nitrosoguanidineinduced gastric carcino-genesis. Fitoterapia., 73(7/8): 604-611.

Velmurugan, B.; Bhuvaneswari, V.; Balasenthil, S. And Nagini, S. (2001). Lycopene, an antioxidant carotenoid modulates glutathione - dependent hepatic bio-transformation enzymes during experimental gastric carcinogenesis. Nutrition Research., 21: 1117-1124.

Vyskocil, A.; Fiala, Z.; Popler, A.; Ettlerova, E. and Emminger, $S$. (1991). The urinary excretion of specific proteins in workers exposed to lead. Arch. Toxicol., 14: 218-221.

Vyskocil, A.; Pancl, J.; Tusl, M.; Ettlerova, E.; Semecky, V.; Kasparova, L.; Lauwerys, R. and 
Bernard, A. (1989). Dose-related proximal tubular dysfunction in male rats chronically exposed to lead. J Appl Toxicol., 9: 395-399.

Wang, C.G., Zhao, Y. and Zheng, R.H. (2006). Effects of tributyltin, benzo[a]pyrene, and their mixture on antioxidant defense systems in Sebastiscus marmoratus. Ecotoxicol Environ Safe., 65:381-387.

Wendelaar Bonga, S.E. (1997). The stress response in Fish. Physiol. Rev., 77: 591- 625 .

Winneke, G.; Collet, W. and Lilienthal, H. (1988). The effects of lead in laboratory animals and environmentally exposed children. Toxicology., 49: 291.

Xianquan, S.; Shi, J.; Kakuda, Y. and Yueming, J. (2005). Review stability of lycopene during processing and storage. J. Med Food, 8:413-422.

Yilmaz, S.; Atessahin, A.; Sahna, E.; Karahan, I. And Ozer, S. (2006). Protective effect of lycopene on adriamycin-induced cardiotoxicity and nephrotoxicity. Toxicology., 218: 164-171.

Yousuf, M. H. A.; El-Shahawi. (1999). Trace metals in Lethrinus lentjan fish from Arabian Gulf: Metal accumulation in Kidney and Heart Tissues. Bull. Environ. Contam. Toxicol., 62 (3): 293-300.

Yu-Hung, L. and Shi-Yun, S. (2005). Dietary vitamin E requirement of grouper, pinephelus malabaricus, at two lipid levels, and their effects on immune responses. Aquaculture, 248: 235- 244.

\section{ARABIC SUMMERY}

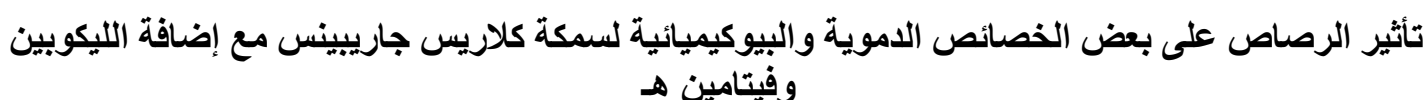
وفيتامين هـ ولهين

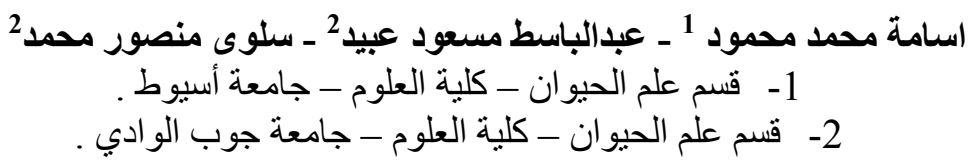

تهدف الدر اسة الحالية إلى بحث التأثير الوقائي للايكوبين وفيتامين هـ ضد التأثيرات الضـارة للإصاص بعد تعرض الأسماك للرصاص لمدة 15 ، 30 يوم .

لقد درست تأثيرات الرصاص بلاصلالة الخصائص الدموية و البيوكيميائية ولقد وجدت زيادة معنوية في نشاط

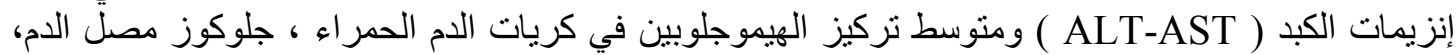

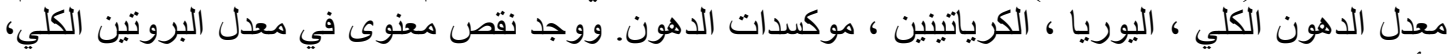

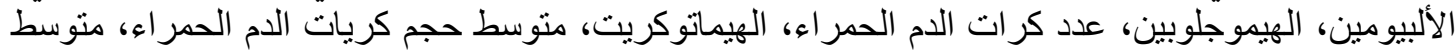

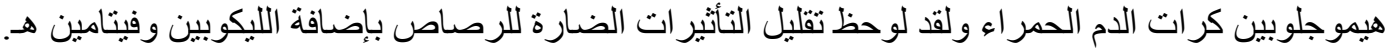

